Universidade de São Paulo

Instituto de Física

\title{
Modelo hierárquico para gases de Coulomb: uma análise via grupo de renormalização
}

\author{
Rafael Jorge Hauy
}

Orientador: Prof. Dr. Domingos Humberto Urbano Marchetti

Dissertação de mestrado apresentada ao Instituto de Física da Universidade de São Paulo, como requisito parcial para a obtenção do título de Mestre em Ciências.

Banca Examinadora:

Prof. Dr. Domingos Humberto Urbano Marchetti - Orientador (IFUSP)

Profa. Dra. Maria Eulália Vares (UFRJ)

Prof. Dr. Gastão de Almeida Braga (UFMG) 
FICHA CATALOGRÁFICA

Preparada pelo Serviço de Biblioteca e Informação do Instituto de Física da Universidade de São Paulo

Hauy, Rafael Jorge

Modelo hierárquico para gases de Coulomb: uma análise via grupo de renormalização. São Paulo, 2021.

Dissertação (Mestrado) - Universidade de São Paulo, Instituto de Física, Depto. de Física Geral.

Orientador: Prof. Dr. Domingos Humberto Urbano Marchetti

Área de Concentração: Física Matemática

Unitermos: 1. Mecânica estatística clássica; 2. Física matemática; 3. Estatística dos gases.

USP/IF/SBI-020/2021 


\title{
University of São Paulo \\ Physics Institute
}

\section{Hierarchical model for Coulomb gases: a renormalization group analysis}

\author{
Supervisor: Prof. Dr. Domingos Humberto Urbano Marchetti
}

Dissertation submitted to the Physics Institute of the University of São Paulo in partial fulfillment of the requirements for the degree of Master of Science.

Examining Committee:

Prof. Dr. Domingos Humberto Urbano Marchetti - Supervisor (IFUSP)

Profa. Dra. Maria Eulália Vares (UFRJ)

Prof. Dr. Gastão de Almeida Braga (UFMG)

São Paulo

2021 



\section{Dedicatória}

À Olizinha, que nos trouxe tanta alegria. 



\section{Agradecimentos}

Três anos se passaram desde o começo do projeto e deste texto. Foi um trabalho divertido, mas também difícil e sua conclusão não teria sido possível sem a grande ajuda que recebi.

Gostaria assim de agradecer: meu orientador, Domingos Marchetti, que desde a iniciação científica tem me guiado, com muita paciência e sabedoria, pelos caminhos da mecânica estatística e da física matemática; à Mari, meu amor, que tanto me apoiou nas dificuldades, além de ter ajudado com a programação e as imagens deste trabalho; aos meus pais, que sempre me incentivaram a continuar com o mestrado e cuidaram tão bem da Olívia enquanto eu trabalhava, ao meu irmão, que além de me ajudar com a dor nas costas também ajudou a cuidar da Olivia; à minha irmã, que ouviu as minhas reclamações; aos meus sogros, que cuidaram da Olívia enquanto eu pesquisava e fazia reuniões; ao Willy, que sempre esteve lá para falar de matemática comigo; aos meus amigos da salinha, que me acompanham e me carregam desde a graduação; aos meus amigos Jão, Frank, Marina e Ana Carolina, que me fizeram companhia nos momentos difíceis do projeto; e à Olívia, que me trouxe tanta luz na vida. A todos vocês, meu mais sincero obrigado.

Por fim gostaria de agradecer à $\mathrm{CNPq}$ pelo financiamento dos dois primeiros anos do projeto. $\mathrm{O}$ presente trabalho foi realizado com apoio da Coordenação de Aperfeiçoamento de Pessoal de Nível Superior Brasil (CAPES) - Código de Financiamento 001. 



\section{Resumo}

Neste trabalho consideramos o modelo do gás de Coulomb de uma espécie, em que as interações foram substituidas por uma decomposição binária com aproximação hierárquica entre os subcubos, para $n$ partículas em um hipercubo unitário de $d$ dimensões. Investigamos o sistema de $n$ partículas em um regime assintótico para $n$ grande no contexto dos grupos de renormalização e procuramos um ponto fixo, da forma $V(n, \beta) e^{-r(\beta)(n-\bar{n})^{2}+b(\beta)(n-\bar{n})}$ para as equações encontradas, onde $\bar{n}$ denota o número de partículas de um estado fundamental e $V$ é uma função periódica, com período $2^{d}$. Com as análises feitas encontramos uma equação que relaciona as escalas do modelo por uma convolução

$$
\tilde{M}_{n}=\sum_{m_{1}=-\infty}^{\infty} \sum_{m_{2}=-\infty}^{\infty} \cdots \sum_{m_{k-1}=-\infty}^{\infty} e^{-r\left(\beta^{\prime}\right)\left(\left(n-m_{1}\right)^{2}+\sum_{i=1}^{k-2}\left(m_{i}-m_{i+1}\right)^{2}+m_{k-1}^{2}\right)}
$$

Essa convolução tem um caráter oscilatório, que podemos observar aplicando a fórmula de Poisson nas convoluções, resultando em

$$
\tilde{M}_{n}=\sqrt{\frac{\left(\pi / r\left(\beta^{\prime}\right)\right)^{k-1}}{k}} \sum_{\xi \in \mathbb{Z}^{k-1}} e^{-\frac{\pi^{2}}{r}\left(\xi, \tilde{J}_{k-1}^{-1} \xi\right)} e^{-2 \pi i(n-\alpha) \sum_{j=1}^{k-1} \frac{j}{k} \xi_{j}}
$$

Exploramos também o comportamento de um ponto fixo gaussiano, mostrando que nessa classe de funções ele é estável.

Palavras-chave: Rigidez; Grupo de Renormalização, Modelo Hierárquico; Ponto Fixo 



\section{Abstract}

In the following work, we consider the single-species Coulomb gas, in which the particle interactions are substituted by a binary hierarchical approximation of subcubes, for $n$ particles in a $d$-dimensional hypercube. We investigate the $n$ particle system for the asymptotic regime (large $n$ ), in the context of renormalization groups. We look for fixed points of the form $V(n, \beta) e^{-r(\beta)(n-\bar{n})^{2}+b(\beta)(n-\bar{n})}$ for the renormalization group equations, where $\bar{n}$ stands for the number of particles in a ground state and $V$ is a periodic function, with period $2^{d}$. The analysis leads to an equation, which relates subsequent scales of the problem by convolutions

$$
\tilde{M}_{n}=\sum_{m_{1}=-\infty}^{\infty} \sum_{m_{2}=-\infty}^{\infty} \cdots \sum_{m_{k-1}=-\infty}^{\infty} e^{-r\left(\beta^{\prime}\right)\left(\left(n-m_{1}\right)^{2}+\sum_{i=1}^{k-2}\left(m_{i}-m_{i+1}\right)^{2}+m_{k-1}^{2}\right)}
$$

This convolution behaves as an oscillation. We can observe it by applying the Poisson summation formula and obtaining

$$
\tilde{M}_{n}=\sqrt{\frac{\left(\pi / r\left(\beta^{\prime}\right)\right)^{k-1}}{k}} \sum_{\xi \in \mathbb{Z}^{k-1}} e^{-\frac{\pi^{2}}{r}\left(\xi, \tilde{J}_{k-1}^{-1} \xi\right)} e^{-2 \pi i(n-\alpha) \sum_{j=1}^{k-1} \frac{j}{k} \xi_{j}} .
$$

We also explore the behaviour of a gaussian fixed point, and show that they are stable in this class of functions.

Key-words: Rigidity; Renormalization Group, Hierarchical Model; Fixed Point 



\section{Sumário}

\begin{tabular}{lll}
\hline 1 & Introdução & 5
\end{tabular}

2 Gás de Coulomb Hierárquico $\quad 11$

2.1 Densidade de Probabilidade . . . . . . . . . . . . . . . . . . . 11

2.2 A Energia . . . . . . . . . . . . . . . . . . . . . . 13

2.3 A Energia Pode Ser Truncada . . . . . . . . . . . . . . . . . . . . . . 16

2.4 Fórmula de Recorrência para a Energia $\ldots \ldots \ldots \ldots \ldots$. . . . . . . . . 17

2.5 Mínima Energia $\ldots \ldots \ldots \ldots \ldots \ldots \ldots \ldots \ldots$

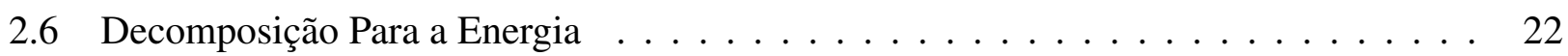

3 Gás de Coulomb Hierárquico com Background 25

3.1 Grupo de Renormalização -Relações de Recorrência . . . . . . . . . . . . . . . . . 26

3.2 Função de Partição . . . . . . . . . . . . . . . . . . . . . . . 26

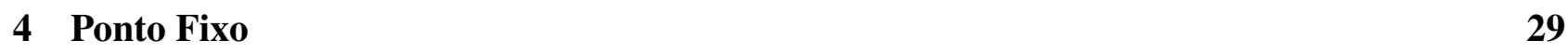

$4.1 \quad$ Ansatz $\ldots \ldots \ldots \ldots \ldots \ldots \ldots$

4.2 A Convolução Contínua . . . . . . . . . . . . . . . . . . . . . . . . 34

4.3 Maior Contribuição da Soma . . . . . . . . . . . . . . . . . . . . 36

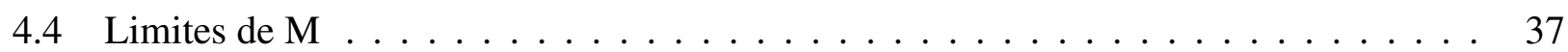

4.5 A Fórmula de Poisson $\ldots \ldots \ldots \ldots \ldots \ldots \ldots \ldots$

$4.6 \quad$ Caracterizando as Oscilações $\ldots \ldots \ldots \ldots \ldots \ldots \ldots$. . . . . . . . . . . . . . 41

4.7 Limite de r pequeno $\ldots \ldots \ldots \ldots \ldots \ldots \ldots \ldots$. . . . . . . . . . . . . . 42

4.8 Estabilidade do ponto fixo no limite de altas temperaturas $\ldots \ldots \ldots$. . . . . . 43

\begin{tabular}{|ll|}
\hline A Matriz Q & 45
\end{tabular}

$5.1 \quad$ O Limite de $\mathrm{n}$ grande em $\mathrm{Q} \ldots \ldots \ldots \ldots \ldots \ldots \ldots \ldots$

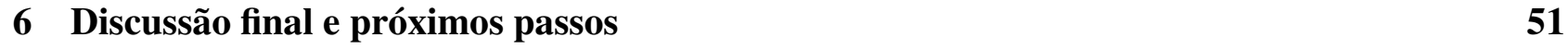

\begin{tabular}{|l|l}
\hline Appendices & 53
\end{tabular} 
\begin{tabular}{|lr}
\hline A Integrais & 55
\end{tabular}

\begin{tabular}{|cc}
\hline B Delta de Dirac & 57
\end{tabular}

\begin{tabular}{|lr|}
\hline C Fórmula de Poisson & 59
\end{tabular} 


\section{Capítulo 1}

\section{Introdução}

Quando se pensa em processos estocásticos de partículas, uma das principais e primeiras quantidades que vêm à mente é o número de partículas $N(U)$ em um dado domínio $U$ e sua flutuação, geralmente medida pela $\operatorname{Var}(N(U))$. Essas são grandezas importantes, pois governam as distribuições de probabilidade de diversas outras quantidades, muitas vezes todas.

Não faltam exemplos de modelos em que as flutuações de $N(U)$ são ditas extensivas, i.e. assintoticamente crescem como o volume $|U|$ da partição em questão (como os modelos de sistemas de partículas citados por Ghosh e Lebowitz [13], como processos de Poisson, de Gibbs, Bosonicos e outros); mas isso nem sempre é verdade. Por exemplo, para o limite termodinâmico de sistemas Coulombianos e autovalores de matrizes aleatórias, as flutuações são sub-extensivas e, em muitos casos, a variância cresce governada por $|\partial U|$, que denota a área euclideana da fronteira $\partial U$ do domínio. Processos pontuais com flutuações sub-extensivas são ditos hiperuniformes. Ginibre, por exemplo, deduz uma condição suficiente, para que a razão $\frac{\operatorname{Var}(N(U))}{N(U)}$ seja finita e estritamente positiva [10] no caso de gases de uma espécie que satisfaçam a expansão de Mayer.

Em Torquato e Stillinger [11], os autores mostram ainda que para qualquer sistema de pontos interagentes que tenha um padrão estatísticamente homogêneo e isotrópico, a $\operatorname{Var}(N(U))$ não pode crescer mais devagar do que $|\partial U|$. Uma questão levantada em seu texto é ainda: para que classe de padrões pontuais o crescimento da variância é da ordem exata da superfície do domínio? Para esse tipo de sistema é dado o nome de rígido. Um pouco de intuição sobre o assunto pode ser encontrada na figura 2 de [8].

Ainda de acordo com Torquato-Stillinger, poucos padrões foram mostrados rigorosamente serem rigidos, mas em um desenvolvimento recente da área, Chatterjee [6] consegue estimativas para $\operatorname{Var}(N(U))$ em um modelo Coulombiano hierárquico nos limites microscópicos e mesoscópicos. Essas estimativas são importantes para determinar a rigidez de $N(U)$. Em seu trabalho, Chatterjee considera um gás Coulombiano de uma espécie interagindo via potencial hierárquico, uma simplificação do potencial Coulombiano, que considera interações em blocos das partículas do sistema. 
No trabalho de Chatterjee, a probabilidade conjunta de que um elemento qualquer de uma partição com $n$ partículas tenha suas 8 subpartições $v_{i}$ com exatamente $n_{i}$ partículas, é dado por:

$$
\mathbb{P}\left(N\left(v_{i}\right)=n_{i} \text { para } i=1, \ldots, 8\right)=8^{-n} \frac{n !}{n_{1} ! \ldots n_{8} !} e^{-\beta \sum_{1 \leq i \neq j \leq 8} n_{i} n_{j}} \frac{\prod_{i=1}^{8} Z\left(2 \beta, n_{i}\right)}{Z(\beta, n)}
$$

Uma estimativa desta quantidade em relação a probabilidade de $N\left(v_{i}\right)=m_{i}, i=1, \ldots, 8$ se encontrar no estado fundamental, definido como $11 m_{1}+\cdots+m_{8}=n$ e $m_{i}-\frac{n}{8} \in\{0,1\}$; é usada para calcular a flutuação $\operatorname{Var}\left(N\left(v_{i}\right)\right)$.

É necessário para isso, estabelecer de que forma a função de partição $Z_{n}$ de um sistema de $n$ partículas se comporta em relação a $\beta$ e $n$. Recentemente Ganguly-Sarkar [8] mostraram que $Z_{n}$ satisfaz

$$
Z_{n}^{G r} \leq Z_{n} \leq Z_{n}^{G r} e^{\log ^{6}(n)}
$$

onde $Z_{n}^{G r}$ é a contribuição para a função de partição devido às configurações no estado fundamental, e concluíram que

$$
\operatorname{Var}\left(N\left(v_{i}\right)\right) \leq K(\beta) \log ^{12} n
$$

Mesmo com esse resultado, os autores não estabelecem qual seria a forma das flutuações do número de partículas nos elementos de uma partição, com isso quero dizer, que tipo de distribuição de probabilidade governa essas flutuações. Entender essas distribuições se traduz, no contexto desse trabalho, em encontrar uma função de partição $Z$ para o sistema. A abordagem que encontramos para isso foi a dos grupos de renormalização, que dão diversas informações sobre $Z$. A escolha dessa filosofia se dá, entre outros motivos, pois potenciais de longo alcance aumentam a quantidade de escalas que precisam ser observadas na análise de um dado problema, ainda mais perto de um ponto crítico, onde não existem comprimentos característicos. A filosofia do grupo de renormalização entra justamente para contornar esse problemas, por meio da divisão do sistemas em partições nas diferentes escalas de tamanho definido, e integrando o sistema em etapas em cada uma das escalas. Como a estrutura do gás se repete identicamente em todas as escalas, as equações se simplificam. Por conta disso, no nosso trabalho colocamos todo o problema dentro do contexto dos grupos de renormalização.

As nossas equações são obtidas considerando a função de partição

$$
Z(\beta, n)=\sum_{\substack{0 \leq n_{1}, \ldots, n_{8} \leq n \\
\sum_{i} n_{i}=n}}\left(\begin{array}{c}
n \\
n_{1} \ldots n_{8}
\end{array}\right) e^{-\beta \sum_{1 \leq i \neq j \leq 8} n_{i} n_{j}} \prod_{i=1}^{8} 8^{-n_{i}} Z\left(2 \beta, n_{i}\right)
$$

\footnotetext{
${ }^{1}$ Veja a definição de estado fundamental na seção "Mínima Energia"
} 
mas com uma pequena reformulação

$$
W(\beta, n):=\frac{e^{\frac{\beta}{2} n^{2}}}{n !} Z(\beta, n)
$$

e essa nova quantidade é o que nos permite considerar a equação (1.4). Observamos que o somando de (1.4), quando dividido por $Z(\beta, n)$ coincide, precisamente, com a distribuição conjunta de probabilidade (1.1). A nossa versão tem uma forma que explicita características da função $W$ e nos permite compreender melhor seu comportamento assintótico para $n$ grande

$$
W(\beta, n)=\sqrt{\frac{\pi^{k-1}}{k r^{k-1}}} e^{-\frac{\beta}{2} n^{2}}(W(2 \beta, \cdot) * \cdots * W(2 \beta, \cdot))(n),
$$

onde

$$
\begin{gathered}
f * g(n)=\sum_{0 \leq k \leq n} f(k) g(n-k) \\
r=r(\beta)
\end{gathered}
$$

indica o produto de convolução de duas sequências $f$ e $g$ e temos $k$ termos na convolução de (1.6).

A análise que fazemos nesse trabalho para a solução assintótica da equação (1.6) se encaminha na direção de mostrar que a probabilidade de $N\left(v_{i}\right)$ desviar de seu valor no estado fundamental $m_{i}$, denotado nesta dissertação por $\bar{n}_{i}$, pode ser escrita como

$$
e^{-r(\beta)\left(n_{i}-\bar{n}_{i}\right)^{2}} V\left(n_{i}\right)
$$

em que $r$ é uma uma função estritamente positva e $V$ é uma função periódica de período 8 (no caso tridimensional de Chatterjee). Essa afirmação ainda não está finalizada, mas nosso estudo se encaminha nessa direção.

Se essa afirmação for verdadeira, conseguiremos demonstrar, em um trabalho futuro, estimativas mais justas do que as obtidas em [6] e [8] de limite superior para as $\operatorname{Var}\left(N\left(v_{i}\right)\right)$.

Além da diferença de abordagem na análise do problema, apresentamos um modelo considerado o mais simples de gases de Coulomb, o "Jellium", que é um plasma de uma componente. O Jellium consiste em partículas com uma carga $e$ positiva se movendo em um background fixo de carga negativa e densidade - $\rho$ e. Nos trabalhos de Chatterjee [6] e Ganguly-Sarkar [8], os autores também consideram um plasma de uma componente, mas sem o background uniforme. Acrescentar o background nos trará a vantagem de não termos de considerar degenerescência no estado fundamental, bastando apenas um representante, $\operatorname{com} \bar{n}_{i}, i=1, \ldots, 2^{d}$ satisfazendo entre gerações as mesmas relações no parágrafo seguinte a 1.1 satisfeitas por $\left\{m_{i}\right\}$, dessa classe de configurações.

Nosso objetivo final é realizar um estudo via grupo de renormalização dos fenômenos de rigidez e hiperuniformidade e para isso tentamos, neste trabalho, entender qual é a forma assintótica no número de partículas da função de partição do sistema. Esses dois fenômenos são definidos da seguinte forma: 
Definição 1. Se um processo aleatório pontual tem a propriedade que sua flutuação cresce mais lentamente que $\sqrt{n}$, então é denominado rígido. A rigidez diz respeito à determinação quase certa de quantidades estatísticas em um domínio $U$, dada somente a configuração de partículas fora do domínio (ou seja em $U^{c}$ ).

Definição 2. Hiper-uniforme é um processo pontual aleatório sub extensivo em uma região, ou seja, que tem o crescimento de $\operatorname{Var}(n)$ no domínio $U$ proporcional a algo menor que o volume da região.

Em suma, seguindo as ideias de Chatterjee [6] e Ganguly-Sarkar [8], analisamos o estado fundamental do gás e esperamos que ele seja o que mais contribui para a função de partição. A grande diferença da nossa análise é que colocamos o problema dentro do contexto de grupos de renormalização, olhando a transformação introduzida por Marchetti-Perez [2] no contexto da transição de KosterlitzThouless, e assim tiramos mais informações sobre a função de partição. Um indício de que devemos olhar para flutuações Gaussianas é o trabalho de Jancovici, Lebowitz e Magnificat [4], que estuda também o Jellium e estima as distribuições de probabilidade das flutuações de carga em grandes domínios esféricos em três dimensões e determina que a flutuação é Gaussiana quando a quantidade de cargas for uma ordem menor que a superfície do domínio. Uma revisão dos resultados pode ser encontrada no artigo de Ghosh-Lebowitz de 2017 [13].

Por fim, damos aqui uma visão geral de como o trabalho se organiza: começando no segundo capítulo, definimos o nosso modelo de $n$ partículas em $d$ dimensões, confinadas em uma caixa de comprimento unitário e encontramos expressões para a energia em regiões do sistema; também caracterizamos o estado fundamental do sistema. Ele é caracterizado por ter uma diferença de, no máximo, uma partícula entre quaisquer dois elementos de partições de uma hierarquia. Também encontramos uma equação que relaciona a energia $\mathcal{H}_{n_{D_{0}}}$ de um elemento $D_{0}$ de uma partição do sistema, que contém $n_{D_{0}}$ partículas; com as energias $\mathcal{H}_{n_{D_{q}}}$ de cada elemento $D_{q}$ de suas subpartições, que contém $n_{D_{q}}$ partículas

$$
\sum_{D_{q}} \mathcal{H}_{n_{D_{q}}}+2^{(d-2)}\left(\begin{array}{c}
n_{D_{0}} \\
2
\end{array}\right)=\mathcal{H}_{n_{D_{0}}}+\left(\begin{array}{c}
n_{D_{0}} \\
2
\end{array}\right)
$$

onde $\sum_{D_{q}}$ denota a soma da energia em cada elemento de uma subpartição de $D_{0}$.

No terceiro capítulo, introduzimos um background de cargas negativas, distribuidas como em um estado fundamental caracterizado anteriormente e encontramos as relações de recorrência que caracterizam o grupo de renormalização do sistema, i.e., tendo a expressão para as energias, podemos encontrar uma relação de recorrência para as funções de partição das partições, o que é, efetivamente, uma relação para as medidas de probabilidade do sistema.

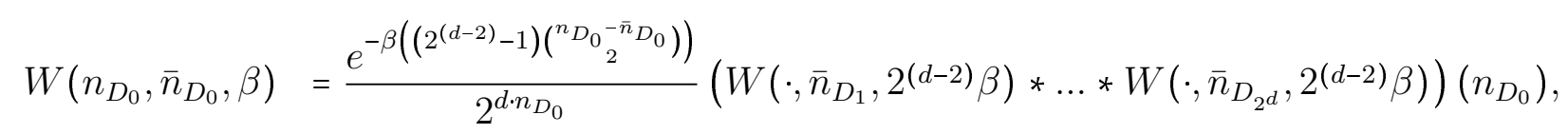

onde $D_{1}, \ldots, D_{2^{d}} \in \mathcal{D}_{1}$ e $\bar{n}$ é a quantidade de partículas negativas do background no hipercubo em questão. 
No quarto capítulo vemos as principais contribuições do trabalho, onde propomos uma forma para o ponto fixo do sistema

$$
W(n, \bar{n}, \beta)=G(n, \bar{n}, \beta) V(n, \beta),
$$

$\operatorname{com} G(n, \bar{n}, \beta):=e^{-r(\beta)(n-\bar{n})^{2}+b(\beta)(n-\bar{n})}$ e $V(n, \beta)$ sendo uma função periódica em $n$, cujo período veremos ser $2^{d}$. Com essa ideia analisamos de que maneira esse ponto fixo se comporta na equação (1.6). Percebemos aqui, primeiro numéricamente, que quando colocamos uma Gaussiana na convolução, isso resulta em uma função da forma $G(n, \bar{n}, \beta) V(n, \beta)$ para todas as temperaturas, onde $G(n, \bar{n}, \beta)$ é uma função Gaussiana em $n$, centrada em $\bar{n}$ e tal que $V(n, \beta)$ é uma função periódica, de período $2^{d}$. Motivados por isso, mostramos que existe um termo que tem uma maior contribuição na convolução e que conforme qualquer variável $m_{i}$ se afasta desse máximo por um fator $\delta$, o valor do termo decai com um fator $\mathcal{O}\left(\exp \left(-r\left(\frac{1}{k-i}+\frac{1}{i}\right) \delta^{2}\right)\right)$, dessa forma estendemos os limites das somas da convolução:

$$
\tilde{M}_{n}=\sum_{m_{1}=-\infty}^{\infty} \sum_{m_{2}=-\infty}^{\infty} \cdots \sum_{m_{k-1}=-\infty}^{\infty} e^{-r\left(\beta^{\prime}\right)\left(\left(n-m_{1}\right)^{2}+\sum_{i=1}^{k-2}\left(m_{i}-m_{i+1}\right)^{2}+m_{k-1}^{2}\right)}
$$

no lugar de

$$
\sum_{m_{1}=-1}^{n} \cdots \sum_{m_{\alpha}=-1}^{m_{\alpha-1}} \sum_{m_{\alpha+1}=0}^{m_{\alpha}} \cdots \sum_{m_{k-1}=0}^{m_{k-2}} e^{-r\left(\beta^{\prime}\right)\left(\left(n-m_{1}\right)^{2}+\sum_{i=1}^{k-2}\left(m_{i}-m_{i+1}\right)^{2}+m_{k-1}^{2}\right)} .
$$

Essa nova versão aproximada da convolução, nos permite usar a fórmula de Poisson para escrevermos

$$
\tilde{M}_{n}=\sqrt{\frac{\left(\pi / r\left(\beta^{\prime}\right)\right)^{k-1}}{k}} \sum_{\xi \in \mathbb{Z}^{k-1}} e^{-\frac{\pi^{2}}{r}\left(\xi, \tilde{J}_{k-1}^{-1} \xi\right)} e^{-2 \pi i(n-\alpha) \sum_{j=1}^{k-1} \frac{j}{k} \xi_{j}},
$$

e assim mostramos analiticamente que, para valores suficientemente grandes de $n$, as Gaussianas na convolução resultam em termos da forma $G(n, \bar{n}, \beta) V(n, \beta)$.

Ainda no quarto capítulo damos estimativas para as oscilações resultantes da convolução e argumentamos que, no limite de altas temperaturas ( $\beta$ pequeno), podemos desprezar as oscilações. Com essa aproximação calculamos os valores dos expoentes $r$ e $b$ e concluimos que $r$ é um ponto fixo estável. Isso significa que, mesmo que as menores hierarquias do sistema não tenham distribuições tão próximas do ponto fixo (mais ainda dentro da classe de funções gaussianas), as hierarquias maiores, suficientemente distantes, convergem para essa forma, assumindo que a convolução também leva funções da forma periódica, para outras funções da forma periódica. Nos limites de altas temperatras, podemos dizer que essa é a forma do ponto fixo, mas ainda estamos trabalhando em uma forma geral, que valha para todas as temperaturas.

No quinto capítulo escrevemos a convolução de uma forma diferente e com ela encontramos uma relação de grupo de renormalização para a função $V$ em uma subsequência de valores $n$ múltiplos de $2^{d}$, para qualquer temperatura. Por mais que seja só em uma subsequência, estudamos nesse capítulo o que ocorre com as funções periódicas quando submetidas à convolução e mostramos que, de fato, as distribuições do sistema acabam convergindo para a forma proposta pelo Ansatz. 



\section{Capítulo 2}

\section{Gás de Coulomb Hierárquico}

\subsection{Densidade de Probabilidade}

O modelo que tratamos é de um gás interagente de $n$ partículas em um hipercubo de $d>2$ dimensões $D=[0,1)^{d}$, em que as configurações das partículas tem uma densidade de probabilidade conjunta:

$$
\frac{\exp \left(-\beta \sum_{1 \leq i, j \leq n} \omega\left(x_{i}, x_{j}\right)\right)}{Z(n, \beta)}
$$

onde $Z(n, \beta)$ é uma função de normalização da densidade de probabilidade, a função de partição, e $\omega(x, y)$ é um potencial simétrico que se comporta como Coulomb em curtas distâncias. O potencial que vamos tratar é o potencial hierárquico e para descrever esse potencial precisamos do conceito de hierarquias.

Vamos considerar um hiper-cubo $[0,1)^{d}$ com subdivisões diádicas, o que significa que vamos dividir o cubo na metade em todas as dimensões e então dividir as subdivisões. Essas subdivisões são as hierarquias do sistema. Na primeira hierarquia temos $2^{d}$ subcubos de lado $2^{-1}$ e na $k$-ésima hierarquia temos $2^{d k}$ elementos de lado $2^{-k d}$. Essas subdivisões são, a princípio, infinitas. Para evitar problemas de ambiguidade na definição, podemos considerar que, dado um eixo ao longo do qual dividimos ( $x$ por exemplo), a região de um elemento $i$ de uma partição da hierarquia $k$ é definida como $i 2^{d k} \leq x<(i+1) 2^{d k}$. 


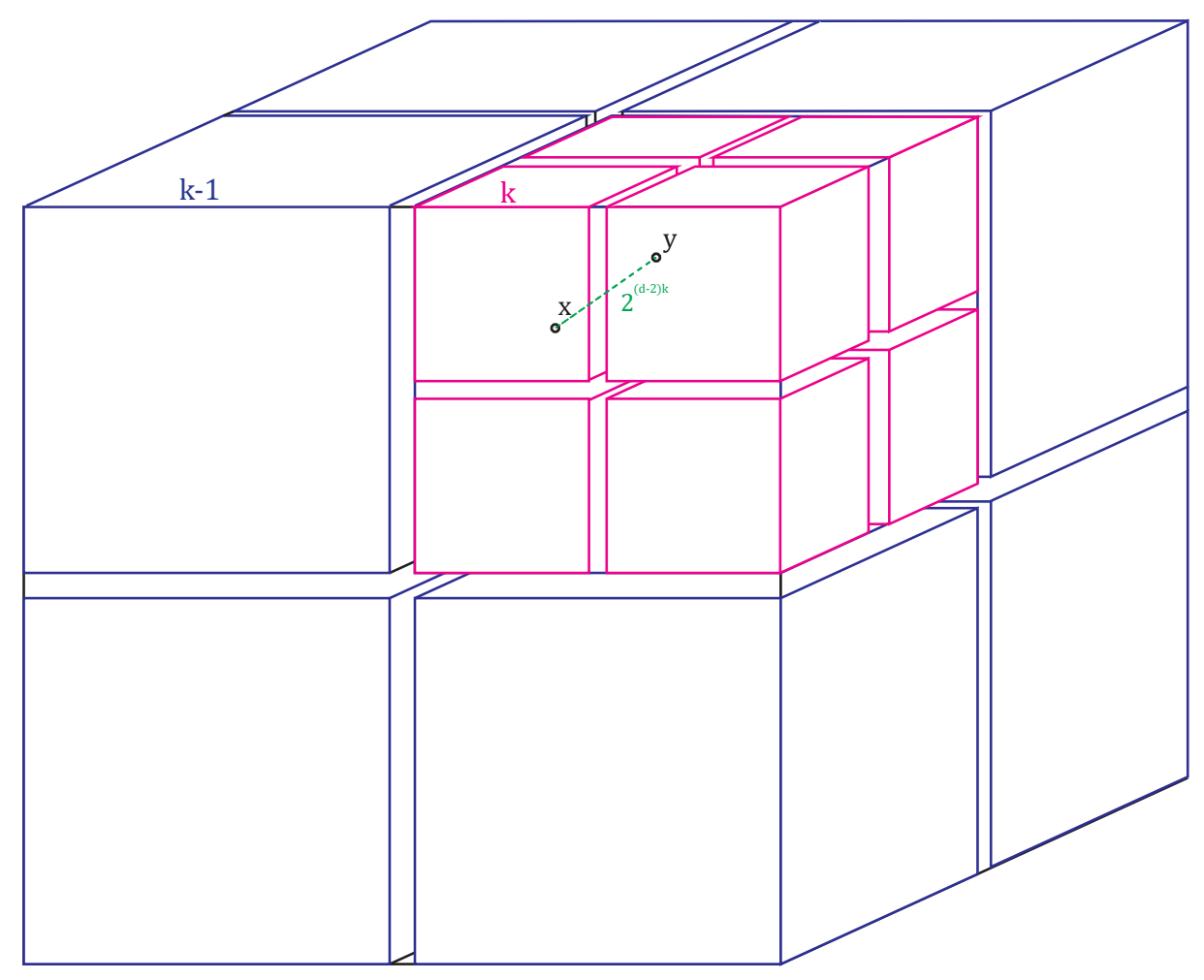

Figura 2.1: Representação do potencial hierárquico.

Definição 3. O potencial hierárquico Coulombiano $\omega(x, y)$ é definido para dois pontos distintos $x e$ y do hipercubo d-dimensional $[0,1)^{d}$ : seja $k=k(x, y)$ a menor hierarquia tal que $x$ e y estejam em dois subcubos distintos nessa hierarquia, então o potencial hierárquico é definido como $w(x, y)=$ $2^{(d-2) k(x, y)}$.

Observação: esse potencial se dá, pois o potencial Coulombiano em $d>2$ entre dois pontos $x$ e $y$ é definido como $\operatorname{dist}(x, y)^{2-d}$ e essa distância $\operatorname{dist}(x, y)$ no modelo hierárquico é $2^{-k(x, y)}$, ou seja, o tamanho da hierarquia que define a distância hierárquica $k$.

Vamos denotar por $\mathcal{D}_{k}$ o conjunto de todos os sub-hipercubos diádicos de $[0,1)^{d}$ de tamanho $2^{-k}$, para algum $d>2$. Em geral vamos denotar por $D_{k}$ um elemento de $\mathcal{D}_{k}$ e notar que so há um elemento $D_{0} \in \mathcal{D}_{0}$ que é o hipercubo inteiro. Definimos ainda o conjunto de todos os subconjuntos diádicos de $[0,1)^{d}$

$$
\mathcal{D}:=\bigcup_{k=0}^{\infty} \mathcal{D}_{k}
$$

Por fim, definimos o conjunto de todas as n-túplas de pontos de $[0,1)^{d}$ como $\Sigma_{n}$ e a energia de uma configuração $\left(x_{1}, \ldots, x_{n}\right) \in \Sigma_{n}$ como

$$
H_{n}\left(x_{1}, \ldots, x_{n}\right):=\sum_{1 \leq i, j \leq n} \omega\left(x_{i}, x_{j}\right)
$$


Assim, a densidade de probabilidade do nosso sistema (2.1) pode ser escrita como

$$
\frac{\exp \left(-\beta H_{n}\left(x_{1}, \ldots, x_{n}\right)\right)}{Z(n, \beta)} .
$$

com

$$
Z(n, \beta):=\int_{[0,1)^{n d}} \ldots \int_{\ln } \exp \left(-\beta H_{n}\left(x_{1}, \ldots, x_{n}\right)\right) d x_{1} \ldots d x_{n}
$$

Definição 4. Definimos configuração como sendo uma disposição de $n_{D}$ partículas

$$
\left.x\right|_{D_{i}}=\left(x_{1}, \ldots, x_{n_{D}}\right)
$$

restritas ao elemento $D$ da partição da escala i e $n_{D}$ como sendo o número de partículas em $D \in \mathcal{D}_{l}$. Em geral, dado $D \in \mathcal{D}_{l},\left.x\right|_{D}$ denota uma configuração e $n_{D}$ sua cardinalidade.

Quando falamos em configuração entende-se uma organização que induza certos números de partículas em determinadas subpartições.

\begin{tabular}{|c|c|c|c|}
\hline & $\bullet$ & & - $\mathrm{D}_{\mathrm{b}}$ \\
\hline • & • & • & ๑ \\
\hline ○ & & $\bullet$ & $\bullet^{D_{d}}$ \\
\hline & & $\bullet$ & • \\
\hline
\end{tabular}

Um exemplo em duas dimensões seria:

$$
\mathrm{D} \in \mathcal{D}_{0}
$$

Nesse exemplo $n_{D}=18, n_{D_{a}}=n_{D_{d}}=6$ e $\left.x\right|_{D_{a}}=\left.x\right|_{D_{d}}$, além disso $n_{D_{b}}=n_{D_{c}}=3$ mesmo com $\left.x\right|_{D_{b}} \neq\left. x\right|_{D_{c}}$.

\subsection{A Energia}

Vamos agora encontrar uma representação diferente para a energia de uma configuração qualquer de $n$ pontos, uma representação que simplifique o sistema. A energia depende das interações dois a 
dois de cada uma das partículas com todas as outras. Nós vamos considerar as interações de $A$ com $B$, mas também as de $B \operatorname{com} A$ e por isso a energia seria duplicada, então as divisões por dois. A principio vamos somar as interações de cada um dos sub-hipercubos de um tamanho $k$, ou seja, todas as interações de elementos de $\mathcal{D}_{k}$ e depois vamos somar sobre todos os tamanhos. Cada hierarquia $\mathcal{D}_{k}$ deve nos dar um potencial de interação $2^{(d-2)(k+1)}$ entre pares distintos de seus membros, vezes o produto da ocupação de cada elemento no par. Dessa forma

$$
H\left(x_{1}, \ldots, x_{n}\right)=\sum_{k=0}^{\infty} 2^{(d-2)(k+1)-1} \sum_{\substack { D \in \mathcal{D}_{k} \\
\begin{subarray}{c}{D^{\prime}, D^{\prime \prime} \\
\in \mathcal{D}_{k+1} \subset D \\
D^{\prime} \neq D^{\prime \prime}{ D \in \mathcal { D } _ { k } \\
\begin{subarray} { c } { D ^ { \prime } , D ^ { \prime \prime } \\
\in \mathcal { D } _ { k + 1 } \subset D \\
D ^ { \prime } \neq D ^ { \prime \prime } } }\end{subarray}} n_{D^{\prime}} n_{D^{\prime \prime}} .
$$

Onde

$$
\sum_{\substack{D^{\prime}, D^{\prime \prime} \\ \in \mathcal{D}_{k+1} \subset D \\ D^{\prime} \neq D^{\prime \prime}}}
$$

significa: a soma sobre todas os elementos $D^{\prime}, D^{\prime \prime} \in \mathcal{D}_{k+1}$, mas tais que ambos estão restritos ao elemento $D$ da partição $\mathcal{D}_{k}$ na escala $k$. Além disso só somamos sobre $D^{\prime} \neq D^{\prime \prime}$.

Para tratar a multiplicação nessa equação vamos notar que, para $D \in \mathcal{D}_{k}$

$$
\begin{gathered}
n_{D}=\sum_{\substack{D^{\prime} \in \mathcal{D}_{k+1} \\
\subset D}} n_{D^{\prime}} \cdot \\
\sum_{\substack{D^{\prime}, D^{\prime \prime} \\
\in \mathcal{D}_{k+1} \subset D \\
D^{\prime} \neq D^{\prime \prime}}} n_{D^{\prime}} n_{D^{\prime \prime}}=n_{D}^{2}-\sum_{\substack{D^{\prime} \in \mathcal{D}_{k+1} \\
\subset D}} n_{D^{\prime}}^{2}
\end{gathered}
$$

e substituir

$$
n_{D}^{2}=2\left(\begin{array}{c}
n_{D} \\
2
\end{array}\right)+n_{D}
$$

como sugerido em [6], na equação anterior para obtermos

$$
\begin{aligned}
& \sum_{\substack{D^{\prime}, D^{\prime \prime} \\
\in \mathcal{D}_{k+1} \subset D \\
D^{\prime} \neq D^{\prime \prime}}} n_{D^{\prime}} n_{D^{\prime \prime}}=n_{D}^{2}-\sum_{\substack{D^{\prime} \in \mathcal{D}_{k+1} \\
\subset D}} n_{D^{\prime}}^{2} \\
&=2\left(\begin{array}{c}
n_{D} \\
2
\end{array}\right)+n_{D}-2 \sum_{\substack{D^{\prime} \in \mathcal{D}_{k+1} \\
\subset D}}\left(\begin{array}{c}
n_{D^{\prime}} \\
2
\end{array}\right)-\underbrace{\sum_{\substack{D^{\prime} \in \mathcal{D}_{k+1} \\
\subset D}} n_{D^{\prime}}}_{n_{D}} \\
& \sum_{\substack{D^{\prime}, D^{\prime \prime} \\
\in \mathcal{D}_{k+1} \subset D \\
D^{\prime} \neq D^{\prime \prime}}} n_{D^{\prime}} n_{D^{\prime \prime}}=2\left(\begin{array}{c}
n_{D} \\
2
\end{array}\right)-2 \sum_{\substack{D^{\prime} \in \mathcal{D}_{k+1} \\
\subset D}}\left(\begin{array}{c}
n_{D^{\prime}} \\
2
\end{array}\right) .
\end{aligned}
$$


Voltando agora para a representação da energia

$$
H\left(x_{1}, \ldots, x_{n}\right)=\sum_{k=0}^{\infty} 2^{(d-2)(k+1)-1} \sum_{D \in \mathcal{D}_{k}}\left(2\left(\begin{array}{c}
n_{D} \\
2
\end{array}\right)-2 \sum_{\substack{D^{\prime} \in \mathcal{D}_{k+1} \\
\subset D}}\left(\begin{array}{c}
n_{D^{\prime}} \\
2
\end{array}\right)\right)
$$

ao invés de somar todos os sub-hipercubos dentro de um hipercubo $D^{\prime} \in \mathcal{D}_{k+1} \subset D$ e somar sobre todos os $D \in \mathcal{D}_{k}$, somamos só sobre todos os sub-hipercubos de uma hierarquia $D^{\prime} \in \mathcal{D}_{k+1}$ :

$$
\begin{gathered}
\sum_{\substack{D \in \mathcal{D}_{k} \\
D^{\prime} \in \mathcal{D}_{k+1} \\
\subset D}}=\sum_{D^{\prime} \in \mathcal{D}_{k+1}} . \\
H\left(x_{1}, \ldots, x_{n}\right)=\sum_{k=0}^{\infty} 2^{(d-2)(k+1)}\left(\sum_{D \in \mathcal{D}_{k}}\left(\begin{array}{c}
n_{D} \\
2
\end{array}\right)-\sum_{D^{\prime} \in \mathcal{D}_{k+1}}\left(\begin{array}{c}
n_{D^{\prime}} \\
2
\end{array}\right)\right) .
\end{gathered}
$$

Essa soma pode ser dividida em duas partes novamente e a variável do segundo termo pode ser renomeada:

$$
\begin{aligned}
& \sum_{k=0}^{\infty} 2^{(d-2)(k+1)}\left(\sum_{D \in \mathcal{D}_{k}}\left(\begin{array}{c}
n_{D} \\
2
\end{array}\right)-\sum_{D^{\prime} \in \mathcal{D}_{k+1}}\left(\begin{array}{c}
n_{D^{\prime}} \\
2
\end{array}\right)\right) \\
& =\left(2^{(d-2)}-1\right) \sum_{k=1}^{\infty} 2^{(d-2)(k)} \sum_{D \in \mathcal{D}_{k}}\left(\begin{array}{c}
n_{D} \\
2
\end{array}\right)+2^{(d-2)}\left(\begin{array}{c}
n_{D_{0}} \\
2
\end{array}\right)
\end{aligned}
$$

onde $D_{0} \in \mathcal{D}_{0}$.

Dessa forma a energia do sistema é

$$
H\left(x_{1}, \ldots, x_{n}\right)=\left(2^{(d-2)}-1\right) \sum_{k=1}^{\infty} 2^{(d-2) k} \sum_{D_{j} \in \mathcal{D}_{k}}\left(\begin{array}{c}
n_{D_{j}} \\
2
\end{array}\right)+2^{(d-2)}\left(\begin{array}{c}
n_{D_{0}} \\
2
\end{array}\right) .
$$

A nova fórmula (2.9) pode ser interpretada como se cada partícula dentro de um subcubo elemento de $\mathcal{D}_{k}$ só interagisse com as partículas dentro do mesmo subcubo, mas com um potencial $\left(2^{(d-2)}-1\right) 2^{(d-2) k}$. Isso ocorre em todas as hierarquias, com um termo corrigindo a primeira. 


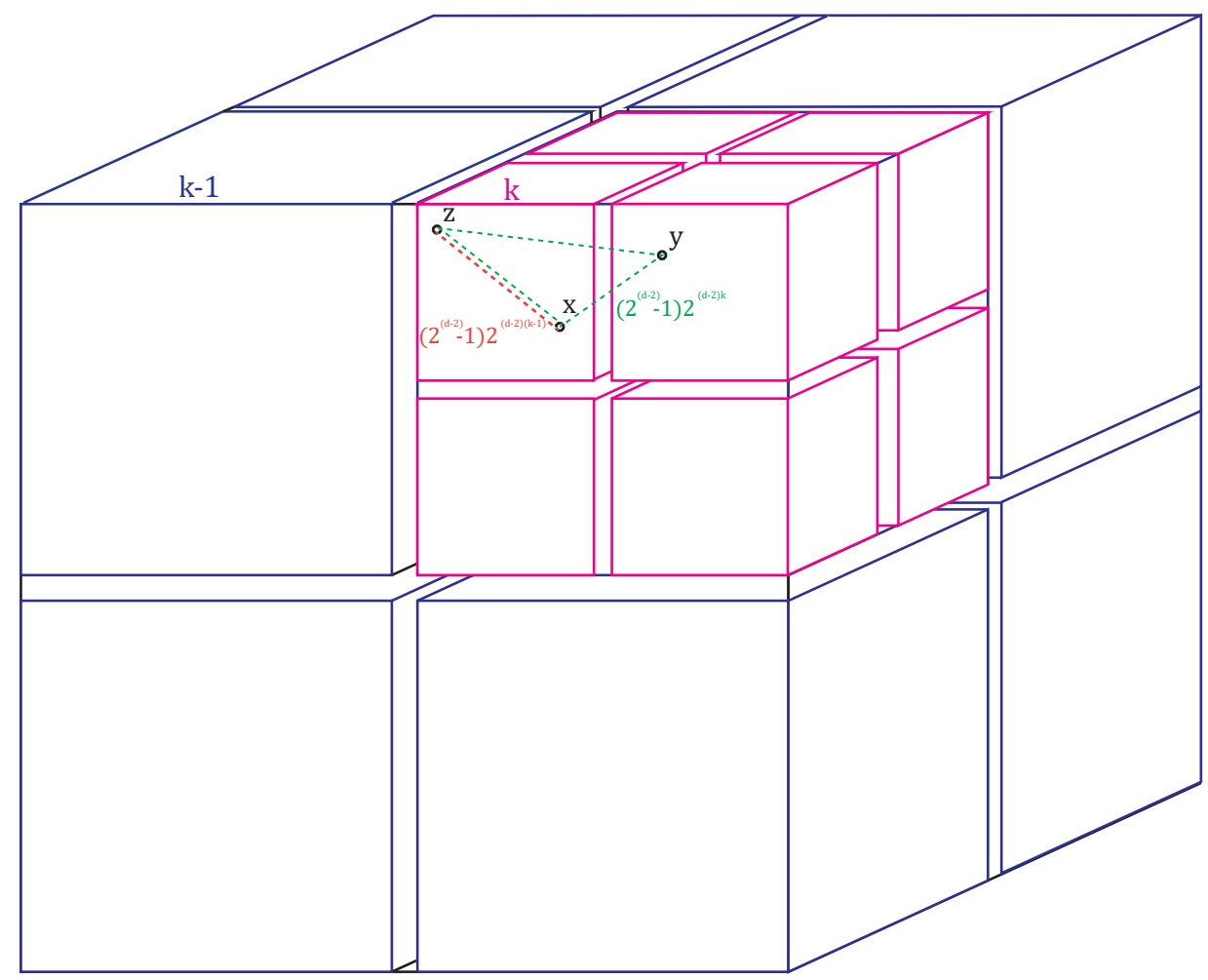

Figura 2.2: Representação da nova interpretação do potencial hierárquico em 3 dimensões.

\subsection{A Energia Pode Ser Truncada}

A introdução da binomial em (2.9) pode ter parecido arbitrária ou mesmo apenas para enfatizar a interpretação de partículas interagentes, mas além disso temos que a introdução do termo linear para que tenhamos a binomial, controla a soma e nos dá uma forma de truncar a soma infinita em $k$.

Para chegar na expressão da energia reescrevemos $n_{D}^{2}$ :

$$
n_{D}^{2}=n_{D}^{2}+\underbrace{n_{D}-n_{D}}_{\text {zero }}=2\left(\begin{array}{c}
n_{D} \\
2
\end{array}\right)+n_{D} .
$$

Esse zero, que permite que escrevamos a expressão em termos de binomiais e é o que regulariza a soma infinita, como veremos.

Definimos uma grandeza $\bar{k}$, que é a menor partição para a qual um sistema não tenha mais de uma partícula por elemento da partição, ou seja,

$$
\bar{k}=\min \left\{k \in \mathbb{N}: n_{D} \in\{0,1\}, \forall D \in \mathcal{D}_{k}\right\}
$$

Para esse $\bar{k}$ e para qualquer $l \geq \bar{k}$ temos que $\left(\begin{array}{c}n_{D_{l}} \\ 2\end{array}\right)=0$, pois $\left(\begin{array}{l}1 \\ 2\end{array}\right)=\left(\begin{array}{l}0 \\ 2\end{array}\right)=0$. Então todas as somas 
$\operatorname{com}\left(\begin{array}{c}n_{D_{l}} \\ 2\end{array}\right)$ podem ser truncadas em $\bar{k}$.

$$
H\left(x_{1}, \ldots, x_{n}\right)=\left(2^{(d-2)}-1\right) \sum_{k=1}^{\bar{k}} 2^{(d-2) k} \sum_{D \in \mathcal{D}_{k}}\left(\begin{array}{c}
n_{D} \\
2
\end{array}\right)+2^{d-2}\left(\begin{array}{c}
n_{D_{0}} \\
2
\end{array}\right) .
$$

\subsection{Fórmula de Recorrência para a Energia}

Tendo uma expressão para a energia total do sistema vamos nos aproximar da filosofia do grupo de renormalização ao encontrar uma outra forma de representar a energia total $H_{n}(x)$. No caso vamos representá-la em termos das energias das subpartições do sistema.

A primeira coisa que temos que fazer é pensar como seria a expressão da energia de um sistema menor, um sistema que consista, por exemplo, de somente um elemento de uma partição $D_{q} \in \mathcal{D}_{Q}$ do nosso sistema original. Essa energia seria

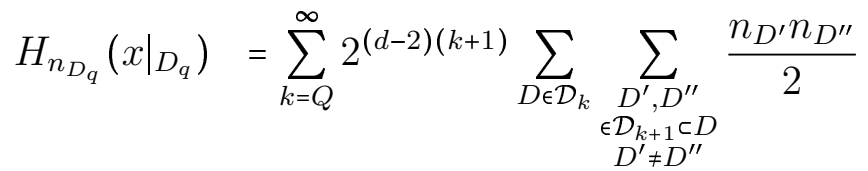

$$
\begin{aligned}
& =\sum_{k=Q}^{\infty} 2^{(d-2)(k+1)}\left(\sum_{D \in \mathcal{D}_{k}}\left(\begin{array}{c}
n_{D} \\
2
\end{array}\right)-\sum_{D^{\prime} \in \mathcal{D}_{k+1}}\left(\begin{array}{c}
n_{D^{\prime}} \\
2
\end{array}\right)\right) \\
& =2^{(d-2)(Q+1)}\left(\begin{array}{c}
n_{D_{q}} \\
2
\end{array}\right)+\sum_{k=Q+1}^{\infty} 2^{k(d-2)} 2^{(d-2)} \sum_{D \in \mathcal{D}_{k}}\left(\begin{array}{c}
n_{D} \\
2
\end{array}\right)-\sum_{k=Q+1}^{\infty} 2^{k(d-2)} \sum_{D \in \mathcal{D}_{k}}\left(\begin{array}{c}
n_{D} \\
2
\end{array}\right) \\
& H_{n_{D_{q}}}\left(\left.x\right|_{D_{q}}\right)=\left(2^{(d-2)}-1\right) \sum_{k=Q+1}^{\bar{k}} 2^{(d-2) k} \sum_{\substack{D \in \mathcal{D}_{k} \\
\subset D_{q}}}\left(\begin{array}{c}
n_{D} \\
2
\end{array}\right)+2^{(d-2)(Q+1)}\left(\begin{array}{c}
n_{D_{q}} \\
2
\end{array}\right) .
\end{aligned}
$$

Podemos derivar essa equação simplesmente refazendo os passos da seção 2.2 , ou ainda lembrando da interpretação que tínhamos estabelecido e escrevendo simplesmente a soma das interações, com potencial $\left(2^{d-2}-1\right) 2^{(d-2) k}$, dentro de cada partição mais uma correção da forma do potencial original nas partículas da maior partição.

A ideia agora é expressar a energia de uma hierarquia em termos da energia da hierarquia seguinte.

$$
\begin{aligned}
& H_{n_{D_{q}}}\left(\left.x\right|_{D_{q}}\right)=\left(2^{(d-2)}-1\right) \sum_{k=Q+1}^{\bar{k}} 2^{(d-2) k} \sum_{\substack{D \in \mathcal{D}_{k} \\
\subset D_{q}}}\left(\begin{array}{c}
n_{D} \\
2
\end{array}\right)+2^{(d-2)(Q+1)}\left(\begin{array}{c}
n_{D_{q}} \\
2
\end{array}\right) \\
& =\left(2^{(d-2)}-1\right) \sum_{k=Q+1}^{\bar{k}} 2^{(d-2) k} \sum_{\substack{D \in \mathcal{D}_{k} \\
\subset D_{q}}}\left(\begin{array}{c}
n_{D} \\
2
\end{array}\right)+\underbrace{\left(2^{(d-2)}-1\right) 2^{(d-2) Q}\left(\begin{array}{c}
n_{D_{q}} \\
2
\end{array}\right)}_{\text {termo } k=Q \text { da soma }}+2^{(d-2) Q}\left(\begin{array}{c}
n_{D_{q}} \\
2
\end{array}\right) \\
& =\left(2^{(d-2)}-1\right) \sum_{k=Q}^{\bar{k}} 2^{(d-2) k} \sum_{\substack{D \in \mathcal{D}_{k} \\
\subseteq D_{q}}}\left(\begin{array}{c}
n_{D} \\
2
\end{array}\right)+2^{(d-2) Q}\left(\begin{array}{c}
n_{D_{q}} \\
2
\end{array}\right) .
\end{aligned}
$$


Definimos ainda um termo para a energia das interações de todos os blocos contidos em um sistema $D_{q} \in \mathcal{D}_{Q}$ :

$$
\mathcal{H}_{n_{D_{q}}}:=\left(2^{(d-2)}-1\right) \sum_{k=Q}^{\bar{k}} 2^{(d-2) k} \sum_{\substack{D \in \mathcal{D}_{k} \\
\subseteq D_{q}}}\left(\begin{array}{c}
n_{D} \\
2
\end{array}\right)
$$

Portanto

$$
H_{n_{D_{q}}}\left(\left.x\right|_{D_{q}}\right)=\mathcal{H}_{n_{D_{q}}}+2^{(d-2) Q}\left(\begin{array}{c}
n_{D_{q}} \\
2
\end{array}\right) .
$$

Ou, usando a expressão (2.12) junto com

$$
\sum_{\substack{D_{p} \in \mathcal{D}_{Q+1} \\
\subseteq D_{q} \in \mathcal{D}_{Q}}} \mathcal{H}_{n_{D_{p}}}=\left(2^{d-2}-1\right) \sum_{k=Q+1}^{\bar{k}} 2^{(d-2) k} \sum_{\substack{D \in \mathcal{D}_{k} \\
\subseteq D_{q} \in \mathcal{D}_{Q}}}\left(\begin{array}{c}
n_{D} \\
2
\end{array}\right)
$$

temos que

$$
H_{n_{D_{q}}}\left(\left.x\right|_{D_{q}}\right)=\sum_{\substack{D_{p} \in \mathcal{D}_{Q+1} \\
\subseteq D_{q} \in \mathcal{D}_{Q}}} \mathcal{H}_{n_{D_{p}}}+2^{(d-2)(Q+1)}\left(\begin{array}{c}
n_{D_{q}} \\
2
\end{array}\right) .
$$

Juntando por fim 2.15) e 2.16

$$
\mathcal{H}_{n_{D_{q}}}=\sum_{\substack{D_{p} \in \mathcal{D}_{Q+1} \\
\subseteq D_{q} \in \mathcal{D}_{Q}}} \mathcal{H}_{n_{D_{p}}}+2^{(d-2) Q}\left(2^{d-2}-1\right)\left(\begin{array}{c}
n_{D_{q}} \\
2
\end{array}\right)
$$

Agora vamos utilizar isso para escrevermos a energia do sistema todo $(Q=0)$ em termos das energias das partições da hierarquia $Q=1$ :

$$
H_{n_{D_{0}}}(x)=\left(2^{(d-2)}-1\right) \sum_{k=1}^{\bar{k}} 2^{(d-2) k} \sum_{D \in \mathcal{D}_{k}}\left(\begin{array}{c}
n_{D} \\
2
\end{array}\right)+2^{(d-2)}\left(\begin{array}{c}
n_{D_{q}} \\
2
\end{array}\right)=\mathcal{H}_{n_{D_{0}}}+\left(\begin{array}{c}
n_{D_{0}} \\
2
\end{array}\right) .
$$

e assim

$$
H_{n_{D_{0}}}(x)=\sum_{D_{q} \in \mathcal{D}_{1}} \mathcal{H}_{n_{D_{q}}}+2^{(d-2)}\left(\begin{array}{c}
n_{D_{0}} \\
2
\end{array}\right)=\mathcal{H}_{n_{D_{0}}}+\left(\begin{array}{c}
n_{D_{0}} \\
2
\end{array}\right)
$$

O que vamos fazer é considerar a relação de recorrência (2.17) de $\mathcal{H}$ daqui em diante.

\subsection{Mínima Energia}

Seguindo as ideias em Ganguly-Sarkar [8], vamos encontrar uma configuração de mínima energia do sistema.

O sistema tem, para cada elemento de uma hierarquia, $2^{d}$ elementos da hierarquia seguinte e a mínima energia se dá quando a distribuição das $n$ partículas é tão uniforme quanto possível em todas as escalas. Isso significa que na maior partição, $\mathcal{D}_{1}$, todos os blocos, exceto por $l=n-\left[\frac{n}{2^{d}}\right] 2^{d}$, tem $\left[\frac{n}{2^{d}}\right]$ partículas, que estão distribuidas uniformemente na primeira partição. Escolhemos $l$ partições 
para terem uma partícula a mais, $\left[\frac{n}{2^{d}}\right]+1$.

Em todas as hierarquias o número de partículas deve ser distribuído dessa forma, para que a energia tenha um mínimo. Isso claramente implica em uma degenerescência do ground state, pois a escolha dos $l$ blocos com partículas a mais pode variar. São $\left(\begin{array}{c}2^{d} \\ l\end{array}\right)$ possibilidades só na primeira hierarquia.

Definição 5. Um estado fundamental ou ground state em um elemento de uma partição $D_{q} \in \mathcal{D}_{Q}$ é uma configuração $\Sigma_{n_{D_{q}}}^{*}$ de $n_{D_{q}}$ partículas tal que a energia $H_{n_{D_{q}}}\left(\Sigma_{n_{D_{q}}}^{*}\right) \leq H_{n_{D_{q}}}\left(\Sigma_{n_{D_{q}}}^{\prime}\right)$ para toda configuração $\sum_{n_{D_{q}}}^{\prime}$ na mesma partição.

Teorema 1. Se uma configuração $\Sigma_{n_{D_{q}}}^{*}$ é um estado fundamental de um elemento de uma partição $D_{q} \in \mathcal{D}_{Q}$, então as configurações $\Sigma_{n_{D_{i}}}^{*}$ dos elementos $D_{i} \in \mathcal{D}_{Q+1}$ da partição $Q+1$ com $D_{i} \subset D_{q}$, $1 \leq i \leq 2^{d}$ são estados fundamentais em suas respectivas partições.

Prova do Teorema 1. Dado que $\Sigma_{n_{D_{q}}}^{*}$ é um estado fundamental, $H_{n_{D_{q}}}\left(\Sigma_{n_{D_{q}}}^{*}\right)$ é um mínimo da energia

$$
H_{n_{D_{q}}}\left(\Sigma_{n_{D_{q}}}^{*}\right)=\sum_{\substack{D_{i} \in \mathcal{D}_{Q_{+1}} \\
D_{i} \subset D_{q}}} \mathcal{H}_{n_{D_{i}}}+2^{(d-2)(Q+1)}\left(\begin{array}{c}
n_{D_{q}} \\
2
\end{array}\right)=\mathcal{H}_{n_{D_{q}}}+2^{(d-2) Q}\left(\begin{array}{c}
n_{D_{q}} \\
2
\end{array}\right)
$$

então todos os $\mathcal{H}_{n_{D_{i}}}$ têm que corresponder a mínimas energias nas partíções $D_{i}$ e, portanto, a estados fundamentais, caso contrário poderíamos escolher uma outra configuração que faria com que as energias, mantendo o mesmo número de partículas em cada elemento das partições, fossem menores e assim contradizendo à hipotese de que $H_{n_{D_{q}}}\left(\Sigma_{n_{D_{q}}}^{*}\right)$ seja um mínimo da energia.

Proposição 1. Considerando as seguintes duas operações

1. Passar $\Delta$ n partículas de um elemento de uma partição $D_{r} \in \mathcal{D}_{Q} \subset D \in \mathcal{D}_{Q-1}$ no estado fundamental para outro elemento $D_{p} \in \mathcal{D}_{Q} \subset D \in \mathcal{D}_{Q-1}$ no estado fundamental com $n_{D_{r}} \leq n_{D_{p}}$, de modo que os dois elementos depois da operação estejam em um estado fundamental (desomogeneizar o sistema).

2. Passar $\Delta$ n partículas de um elemento de uma partição $D_{r} \in \mathcal{D}_{Q} \subset D \in \mathcal{D}_{Q-1}$ no estado fundamental para um elemento $D_{p} \in \mathcal{D}_{Q} \subset D \in \mathcal{D}_{Q-1}$ no estado fundamental com $n_{D_{r}}>n_{D_{p}}$, de modo que depois da operação valha $n_{D_{r}} \geq n_{D_{p}}$ e que os dois elementos depois da operação estejam em um estado fundamental (homogeneizar o sistema).

A primeira aumenta a energia do sistema e a segunda diminui.

Essa proposição nos permite provar um teorema que dá a forma das possíveis configurações do estado fundamental. Após enunciar e provar esse teorema apresentaremos a prova da proposição. 
Teorema 2. Uma configuração $\Sigma_{n_{D_{q}}}^{*}$ é um estado fundamental de um elemento de uma partição $D_{q} \in \mathcal{D}_{Q}$, se e somente se, para as configurações $\Sigma_{n_{D_{i}}}^{*}$ das partições $D_{i}, D_{j} \in \mathcal{D}_{Q+1}$ com $D_{i}, D_{j} \subset D_{q}$, $1 \leq i, j \leq 2^{d}\left|n_{D_{i}}-n_{D_{j}}\right| \leq 1, \forall D_{i}, D_{j} \in \mathcal{D}_{Q+1} \operatorname{com} D_{i}, D_{j} \subset D_{q}$.

Prova do Teorema 2. Com as operações da proposição definidas, percebemos que se um sistema tiver, em uma hierarquia, uma diferença maior que 1 entre as partículas de duas partições quaisquer, podemos sempre realizar a segunda operação para homogeneizar o sistema e assim diminuir sua energia. Dessa forma se um sistema está numa configuração que minimiza a energia, ele não pode ter uma diferença maior que um entre o número de partículas de quaisquer duas partições em uma hierarquia, caso contrário poderíamos realizar a operação 2 e diminuir a energia do sistema, contradizendo a hipótese do estado fundamental.

A volta do teorema consiste em notar que passar partículas de um elemento de uma partição a outro, de modo a aumentar a diferença do número de partículas em caixas de uma mesma hierarquia, aumenta a energia do sistema, enquanto homogeneizar a distribuição diminui a energia. Dessa forma se a diferença for no máximo um entre o número de partículas das partições de uma mesma hierarquia, nenhuma mudança é possível que diminua a energia do sistema e, como as distribuições internas das partições estão em estado fundamentais, agora por hipótese, isso tem que significar que o sistema como um todo está no estado fundamental.

Prova da Proposição 1. A primeira operação aumenta a energia do sistema. Mostrar essa afirmação significa mostrar que

$$
\mathcal{H}_{n_{D_{r}}}+\mathcal{H}_{n_{D_{p}}}<\mathcal{H}_{n_{D_{r}}-\Delta n}+\mathcal{H}_{n_{D_{p}}+\Delta n}
$$

Isso é demonstrado de forma indutiva. Na escala $\mathcal{D}_{\bar{k}-1}$ a energia dentro de um elemento de uma partição é

$$
\begin{gathered}
\mathcal{H}_{n_{D}}=\left(2^{d-2}-1\right) \sum_{k=\bar{k}-1}^{\bar{k}} 2^{(d-2) k} \sum_{\substack{D_{j} \in \mathcal{D}_{k} \\
\subseteq D \in \mathcal{D}_{\bar{k}-1}}}\left(\begin{array}{c}
n_{D_{j}} \\
2
\end{array}\right) \\
=\left(2^{d-2}-1\right)\left[2^{(d-2)(\bar{k}-1)}\left(\begin{array}{c}
n_{D} \\
2
\end{array}\right)+2^{(d-2) \bar{k}} \sum_{i=1}^{2^{d}}\left(\begin{array}{c}
n_{D_{j}} \\
2
\end{array}\right)\right]=\left(2^{d-2}-1\right) 2^{(d-2)(\bar{k}-1)}\left(\begin{array}{c}
n_{D} \\
2
\end{array}\right)
\end{gathered}
$$

já que os $n_{D_{j}}$ são todos 0 ou 1 .

Tendo essa expressão, considerando blocos $D_{r}, D_{p} \in \mathcal{D}_{\bar{k}-1}$ vamos mostrar que

$$
\mathcal{H}_{n_{D_{r}}}+\mathcal{H}_{n_{D_{p}}}<\mathcal{H}_{n_{D_{r}}-\Delta n}+\mathcal{H}_{n_{D_{p}}+\Delta n}
$$

Isso segue diretamente do fato que 


$$
\left(\begin{array}{c}
n_{D_{r}} \\
2
\end{array}\right)+\left(\begin{array}{c}
n_{D_{p}} \\
2
\end{array}\right)<\left(\begin{array}{c}
n_{D_{r}}-\Delta n \\
2
\end{array}\right)+\left(\begin{array}{c}
n_{D_{p}}+\Delta n \\
2
\end{array}\right)
$$

Agora assumimos que essa operação também aumenta a energia se realizada em elementos $D_{1}, D_{2} \in \mathcal{D}_{Q+1} \subset D_{m} \in \mathcal{D}_{Q}$. Dessa forma se $D_{r}, D_{p} \in \mathcal{D}_{Q} \subset D_{M} \in \mathcal{D}_{Q-1}$ estão em estados fundamentais, escrevemos as interações em função das interações de suas subpartições para podermos usar a hipótese indutiva:

$$
\begin{gathered}
\mathcal{H}_{n_{D_{r}}}+\mathcal{H}_{n_{D_{p}}}<\mathcal{H}_{n_{D_{r}}-\Delta n}+\mathcal{H}_{n_{D_{p}}+\Delta n} \\
\sum_{\substack{D_{i} \in \mathcal{D}_{Q+1} \\
\subseteq D_{r} \in \mathcal{D}_{Q}}} \mathcal{H}_{n_{D_{i}}}+2^{(d-2) Q}\left(2^{d-2}-1\right)\left(\begin{array}{c}
n_{D_{r}} \\
2
\end{array}\right)+\sum_{\substack{D_{i} \in \mathcal{D}_{Q+1} \\
\subseteq D_{p} \in \mathcal{D}_{Q}}} \mathcal{H}_{n_{D_{i}}}+2^{(d-2) Q}\left(2^{d-2}-1\right)\left(\begin{array}{c}
n_{D_{p}} \\
2
\end{array}\right) \\
<\sum_{\substack{D_{i} \in \mathcal{D}_{Q+1} \\
\subseteq D_{r}^{\prime} \in \mathcal{D}_{Q}}} \mathcal{H}_{n_{D_{i}}}+2^{(d-2) Q}\left(2^{d-2}-1\right)\left(\begin{array}{c}
n_{D_{r}}-\Delta n \\
2
\end{array}\right)+\sum_{\substack{D_{i} \in \mathcal{D}_{Q+1} \\
\subseteq D_{p}^{\prime} \in \mathcal{D}_{Q}}} \mathcal{H}_{n_{D_{i}}}+2^{(d-2) Q}\left(2^{d-2}-1\right)\left(\begin{array}{c}
n_{D_{p}}+\Delta n \\
2
\end{array}\right)
\end{gathered}
$$

onde $D_{r}^{\prime}$ é $D_{r}$ com $\Delta$ n partículas a menos e $D_{p}^{\prime}$ é $D_{p}$ com $\Delta n$ partículas a mais.

Como

$$
\left(\begin{array}{c}
n_{D_{r}} \\
2
\end{array}\right)+\left(\begin{array}{c}
n_{D_{p}} \\
2
\end{array}\right)<\left(\begin{array}{c}
n_{D_{r}}-\Delta n \\
2
\end{array}\right)+\left(\begin{array}{c}
n_{D_{p}}+\Delta n \\
2
\end{array}\right)
$$

resta mostrarmos que

$$
\sum_{\substack{D_{i} \in \mathcal{D}_{Q+1} \\ \subseteq D_{r} \in \mathcal{D}_{Q}}} \mathcal{H}_{n_{D_{i}}}+\sum_{\substack{D_{i} \in \mathcal{D}_{Q+1} \\ \subseteq D_{p} \in \mathcal{D}_{Q}}} \mathcal{H}_{n_{D_{i}}} \leq \sum_{\substack{D_{i} \in \mathcal{D}_{Q+1} \\ \subseteq D_{r}^{\prime} \in \mathcal{D}_{Q}}} \mathcal{H}_{n_{D_{i}}}+\sum_{\substack{D_{i} \in \mathcal{D}_{Q+1} \\ \subseteq D_{p}^{\prime} \in \mathcal{D}_{Q}}} \mathcal{H}_{n_{D_{i}}}
$$

Passar partículas de $D_{r}$ para $D_{p}$ significa que retiramos algum número de partículas $\delta n$ de $d_{r} \epsilon$ $\mathcal{D}_{Q+1} \subset D_{r}$ e passamos para $d_{p} \in \mathcal{D}_{Q+1} \subset D_{p}$. Repetir esse processo um número finito de vezes com $\delta n$ diferentes corresponde a qualquer troca de partículas $\Delta n$ entre $D_{r}$ e $D_{p}$. Como, por hipótese indutiva, vale a proposição para membros $D_{i} \in \mathcal{D}_{Q+1} \subset D \in \mathcal{D}_{Q}$, sabemos que, por $D_{r}$ e $D_{p}$ estarem em estados fundamentais, as diferenças entre os numeros de partículas nos $D_{i}$ contidos em uma mesma partição são no máximo 1.

Isso significa que só precisamos mostrar que uma troca arbitrária dessas tem que aumentar a energia do sistema, ou seja mostrar que

$$
\mathcal{H}_{n_{d_{r}}}+\mathcal{H}_{n_{d_{p}}} \leq \mathcal{H}_{n_{d_{r}}-\delta n}+\mathcal{H}_{n_{d_{p}}+\delta n}
$$

$O$ sinal de igual na desigualdade corresponde ao caso em que, mesmo com $n_{D_{r}} \leq n_{D_{p}}, n_{d_{r}}+1=$ $n_{d_{p}}$, que é a única forma possível de $n_{d_{r}} \geq n_{d_{p}}$. Nesse caso a igualdade é válida, pois a quantidade de blocos com cada número de partículas que somamos é igual, só mudando de qual elemento da 
partição maior cada um veio. Tendo uma igualdade válida, está provado o passo indutivo nessa situação.

Ignorando então o caso da igualdade, percebemos que, pela hipótese indutiva, passar partículas de modo a aumentar a diferença do número de partículas na escala $\mathcal{D}_{Q+1}$, aumenta a energia do sistema, então a desigualdade é verdadeira. Sabemos disso, pois supusemos que essa passagem de partículas, se feita entre elementos de uma partição contidos em um mesmo $D \in \mathcal{D}_{Q}$ com $d, d^{\prime} \epsilon$ $\mathcal{D}_{Q+1} \subset \mathcal{D}_{Q}$ aumenta a energia, mas a função $\mathcal{H}_{n_{d}}$ depende somente de $n_{d}$ e da distribuição das patrículas dentro de $d$, e não de $D \in \mathcal{D}_{Q}$ que contém $d$. Portanto, numéricamente, vale por nossa hipótese indutiva que

$$
\mathcal{H}_{n_{d}}+\mathcal{H}_{n_{d^{\prime}}}<\mathcal{H}_{n_{d^{-}} \delta n}+\mathcal{H}_{n_{d^{\prime}}+\delta n}
$$

e por esse motivo vale (2.27).

Um detalhe fica faltando ainda: passar partículas de um elemento de uma partição a outro dessa forma pode mudar o $\bar{k}$ do sistema e isso a princípio poderia influenciar de forma negativa a prova, mas na verdade é fácil corrigir esse problema escolhendo, entre as duas situações analisadas, o maior $\bar{k}$ entre elas e assumi-lo com o $\bar{k}$ dos dois casos.

Isso prova que a primeira operação aumenta a energia do sistema.

Para mostrar que a segunda operação diminui a energia do sistema, notamos que ela é o oposto da primeira operação, i.e. se passar partículas de modo a desomogeneizar o sistema aumenta a energia, isso significa que homogeneizar o sistema diminui a energia, pois podemos considerar a segunda operação como sendo um processo que começa com $n_{D}+\Delta n$ partículas em um elemento de uma partição e $n_{D^{\prime}}-\Delta$ n partículas em outro e termina com $n_{D}$ e $n_{D^{\prime}}$ partículas. Todas as desigualdades devem valer da mesma forma e a prova se dá da mesma maneira.

A questão da existência do estado fundamental fica clara, pois temos uma forma exata para um dos estados fundamentais.

\subsection{Decomposição Para a Energia}

Ainda no espírito de caracterizar o estado fundamental, vamos analisar a decomposição da energia de uma configuração em termos da ocupação de partições em escalas sucessivas

$$
\begin{gathered}
H_{n_{D_{q}}}\left(\left.x\right|_{D_{q}}\right)=\mathcal{H}_{n_{D_{q}}}+2^{(d-2) Q}\left(\begin{array}{c}
n_{D_{q}} \\
2
\end{array}\right) \\
H_{n_{D_{q}}}\left(\left.x\right|_{D_{q}}\right)=\sum_{\substack{D_{p} \in \mathcal{D}_{Q+1} \\
\subseteq D_{q} \in \mathcal{D}_{Q}}} \mathcal{H}_{n_{D_{p}}}+2^{(d-2)(Q+1)}\left(\begin{array}{c}
n_{D_{q}} \\
2
\end{array}\right)
\end{gathered}
$$




$$
\mathcal{H}_{n_{D_{q}}}=\sum_{\substack{D_{p} \in \mathcal{D}_{Q+1} \\
\subseteq D_{q} \in \mathcal{D}_{Q}}} \mathcal{H}_{n_{D_{p}}}+\left(2^{(d-2)}-1\right) 2^{(d-2) Q}\left(\begin{array}{c}
n_{D_{q}} \\
2
\end{array}\right)
$$

No estado fundamental sabemos que as maiores subpartições de uma hierarquia tem $\left[\frac{n_{D_{q}}}{2^{d}}\right]$ ou $\left[\frac{n_{D_{q}}}{2^{d}}\right]+1$ partículas, mais precisamente $c_{q}=n_{D_{q}}-\left[\frac{n_{D_{q}}}{2^{d}}\right]$ partições tem uma partícula a mais e $2^{d}-c_{q}$ não tem. Assim:

$$
\mathcal{H}_{n_{D_{q}}}=c_{q} \mathcal{H}_{\left[\frac{n_{D_{q}}}{2^{d}}\right]+1}+\left(2^{d}-c_{q}\right) \mathcal{H}_{\left[\frac{n_{D_{q}}}{2^{d}}\right]}+\left(2^{(d-2)}-1\right) 2^{(d-2) Q}\left(\begin{array}{c}
n_{D_{q}} \\
2
\end{array}\right)
$$





\section{Capítulo 3}

\section{Gás de Coulomb Hierárquico com Background}

Nessa seção introduzimos a ideia de adicionar ao sistema um background de cargas negativas na forma de partículas distribuidas como em um ground state. Dada a natureza discreta do modelo hierárquico, houve um problema de convergência da função de partição ao tentar adicionar um background contínuo. Dessa forma, tentamos um background que fosse o mais uniforme possível em um modelo discreto: o de partículas negativas distribuidas na forma de um estado fundamental. É importante ressaltar que isso não faz do modelo deste trabalho um sistema Coulombiano multi-componente, pois não temos várias distribuições de partículas com cargas distintas. Temos apenas uma distribuição de cargas positivas e um background fixo de cargas negativas. Com esse background a carga total de um elemento de uma partição é igual ao número de partículas nele menos o número de partículas do background. Chamamos essa quantidade líquida de cargas de $\tilde{n}_{D}:=n_{D}-\bar{n}_{D}$ onde $D \in \mathcal{D}_{k}$ e $\bar{n}_{D_{j}}$ é o número de partículas negativas do background.

O novo sistema tem as mesmas interações do sistema anterior com $\tilde{n}$ no lugar de $n$. Isso porque é a carga total de cada bloco que interage com os blocos vizinhos, então não importa se a carga é positiva para as deduções feitas na seção 2.2, a única coisa para a qual temos que nos atentar é que o termo binomial da nossa formula perde a interpretação de ser o número de combinações 2-a-2 dentro dos elementos de uma partição e passa a ser simplesmente uma notação para

$$
\left(\begin{array}{l}
x \\
2
\end{array}\right)=\frac{x^{2}-|x|}{2}
$$

nos casos em que há mais partículas do background no elemento da partição.

Na seção 2.3 podemos extender a noção de $\bar{k}$ de modo a considerar as partículas do background.

Por fim é interessante notar que a introdução desse background elimina a degenerescência do estado fundamental do sistema como um todo, já que definimos uma distribuição para as partículas negativas. 


\subsection{Grupo de Renormalização -Relações de Recorrência}

Vamos agora buscar uma equação de grupo de renormalização para caracterizar o sistema hierárquico. É mais fácil, no nosso contexto, encontrarmos uma relação de recorrência para as funções de partição, isso é na verdade igual a encontrar uma equação para as medidas. A definição de $Z$ é

$$
Z=\int_{D_{0}} d \vec{x} e^{-\beta H_{n}(\vec{x})}
$$

Nessa expressão temos a desvantagem de que, como as partículas são indistinguíveis, não podemos contar permutações de uma configuração. Como isso dificulta as contas vamos contar as permutações e depois dividir pelo número delas $n !=n_{D_{0}}$ !. Assim

$$
Z=\int_{D_{0}} \frac{d \vec{x} e^{-\beta H_{n}(\vec{x})}}{n !}
$$

\subsection{Função de Partição}

Como a função de partição depende diretamente da energia, fica fácil utilizar a relação de recorrência 2.19 estabelecida para encontrarmos uma relação de recorrência para ela. Essa nova relação que nos será útil para a análise posterior do sistema.

$$
\begin{gathered}
Z=\int_{D_{0}} \frac{d \vec{x}}{n !} e^{-\beta\left(\mathcal{H}_{n_{D_{0}}}+\left({ }^{n_{D_{0}}}{ }_{2}\right)\right)}=\int_{D_{0}} \frac{d \vec{x}}{n !} e^{-\beta\left(\sum_{D_{q} \in \mathcal{D}_{1}} \mathcal{H}_{n_{D_{q}}}+2^{d-2}\left({ }^{n}{ }_{2}\right)\right)} \\
Z=e^{-\beta 2^{d-2}\left({ }^{n} D_{0}\right)} \prod_{D_{q} \in \mathcal{D}_{1}} \int_{D_{0}} \frac{d^{n} x}{n !} e^{-\beta \mathcal{H}_{n}}{ }_{D_{q}}
\end{gathered}
$$

Podemos agora integrar cada conjunto de partículas $\vec{x}$ somente no bloco $D_{q}$ ao qual ele está limitado, mas então precisamos também somar sobre todas as possibilidades de números de partículas que podem haver naquele bloco. Tendo em mente que a soma do número de partículas em todos os blocos tem que ser, ao final, $\sum_{q} n_{D_{q}}=n_{D_{0}}$, a função de partição se torna

$$
Z=e^{-\beta 2^{d-2}\left({ }^{n} D_{0}\right)} \sum_{\substack{\left\{n_{D_{q}}\right\} \\ \sum_{q} n_{D_{q}}=n_{D_{0}}}} \prod_{D_{q} \in \mathcal{D}_{1}} \int_{D_{q}} \frac{d^{n_{D_{q}} x}}{n_{D_{q}} !} e^{-\beta \mathcal{H}_{n_{D_{q}}}}
$$

Igualando essa expressão com a expressão (3.2) original de $Z$, temos que

$$
Z=\int_{D_{0}} \frac{d^{n} x}{n !} e^{-\beta\left(\mathcal{H}_{n_{D_{0}}}+\left({ }^{n_{D_{0}}}\right)\right)}=e^{-\beta 2^{d-2}\left({ }^{n} D_{2}\right)} \sum_{\substack{\left\{n_{D_{q}}\right\} \\ \sum_{q} n_{D_{q}}=n_{D_{0}}}} \prod_{D_{q} \in \mathcal{D}_{1}} \int_{D_{q}} \frac{d^{n_{D_{q}}} x}{n_{D_{q}} !} e^{-\beta \mathcal{H}_{n_{D_{q}}}}
$$




$$
\int_{D_{0}} \frac{d^{n} x}{n !} e^{-\beta \mathcal{H}_{n}}=e^{-\beta\left(2^{d-2}-1\right)\left(\begin{array}{c}
n_{D_{0}} \\
2
\end{array}\right)} \sum_{\substack{\left\{n_{D_{q}}\right\} \\
\sum_{q} n_{D_{q}}=n_{D_{0}}}} \prod_{D_{q} \in \mathcal{D}_{1}} \int_{D_{q}} \frac{d^{n_{D_{q}}} x}{n_{D_{q}} !} e^{-\beta \mathcal{H}_{n}}{ }_{D_{q}}
$$

É possível notar uma grande semelhança entre as integrais de ambos os lados da igualdade acima. Essa semelhança nos leva a definir uma nova função $W$, que está relacionada com a função de partição, e realizaremos nossas contas agora com essa função, para a qual é mais simples encontrar uma relação de recorrência.

$$
W\left(\tilde{n}_{D_{0}}, \beta\right):=\int_{D_{0}} \frac{d^{n} x}{n !} e^{-\beta \mathcal{H} \tilde{n}_{D_{0}}}=\frac{Z}{n !} e^{\beta\left(\tilde{n}_{2}^{\tilde{n}_{0}}\right)}
$$

Do lado direito de (3.3) temos quase a função $W$, mas com uma integral sobre $D_{q}$ somente e não $D_{0}$. Seguindo a filosofia do grupo de renormalização, realizamos uma mudança de escala no sistema para integrá-lo em $D_{0}$. Então a ideia é que expandamos o hipercubo $D_{q} \in \mathcal{D}_{1}$ com tudo o que ele contém, para ficar do tamanho de $D_{0}$, mas corrigindo as interações para que elas tenham a mesma intensidade, apesar da mudança nas escalas.

Dessa forma a energia vai se tornar $H_{n_{D_{0}}}\left(\left.x^{\prime}\right|_{n_{D_{q}}}\right)$, onde $\left.x^{\prime}\right|_{n_{D_{q}}}$ representa as partículas que estavam no elemento $D_{q}$ da partição de ordem $Q$ do sistema original, agora reescaladas para que ocupem um sistema do tamanho $D_{0}$. Isso é equivalente à energia de um sistema com $n_{D_{q}}$ partículas, dispostas como as partículas reescaladas, portanto, consideramos a energia desse sistema reescalado como $H_{n_{D_{q}}}(\vec{x})$ e o elemento de integração $\left.d x\right|_{D_{q}}=\left.\frac{1}{2^{d}} d x\right|_{D_{0}}$.

Por último precisamos considerar que as interações do potencial mudam com a distância, então quando reescalamos precisamos do potencial da hierarquia original, ou seja $2^{(d-2)}$ vezes o potencial da hierarquia do sistema reescalado. Por isso multiplicamos os termos dentro da integral que dependem do potencial por $2^{(d-2)}$.

Assim nossa expressão 3.3 fica

$$
\begin{aligned}
W\left(\tilde{n}_{D_{0}}, \beta\right) & =e^{-\beta\left(2^{d-2}-1\right)\left({ }^{n} D_{0}\right)} \sum_{\substack{\left\{n_{D_{q}}\right\} \\
\sum_{q} n_{D_{q}}=n_{D_{0}}}} \prod_{D_{q} \in \mathcal{D}_{1}} \frac{1}{2^{d \cdot n_{D_{q}}}} \underbrace{\int \frac{d^{n_{D_{q}}} x}{n_{D_{q}} !} e^{-\beta 2^{d-2} \mathcal{H}_{\tilde{n}_{D_{q}}}}}_{D_{D_{0}}} \\
& =e^{-\beta\left(2^{d-2}-1\right)\left({ }^{n}{ }_{D_{0}}, 2^{(d-2)} \beta, x\right)} \sum_{\substack{\left\{n_{D_{q}}\right\} \\
\sum_{q} n_{D_{q}}=n_{D_{0}}}} \frac{1}{2^{d \cdot n_{D_{0}}}} \prod_{D_{q} \in \mathcal{D}_{1}} W\left(\tilde{n}_{D_{q}}, 2^{(d-2)} \beta\right) .
\end{aligned}
$$

Definição 6. para duas funções $f, g: \mathbb{N} \mapsto \mathbb{C}$ a convolução discreta de $f$ e g é dada por:

$$
f * g(n):=\sum_{m=0}^{n} f(m) g(n-m)=\sum_{\substack{l, m=0 \\ l+m=n}}^{n} f(l) g(m)
$$


quando as funções só admitem valores de $m>0$, ou ainda podemos estender essa definição para

$$
f * g(n):=\sum_{m=-\infty}^{\infty} f(m) g(n-m)=\sum_{\substack{l, m=-\infty \\ l+m=n}}^{\infty} f(l) g(m)
$$

que será usada no contexto da fórmula de Poisson em breve.

Podemos notar aqui, que a função $W\left(\tilde{n}_{D_{0}}, \beta\right)$ está sendo somada apenas no número de partículas, e não no background. Dessa forma a nossa definição de convolução claramente se aplica à conta se denotarmos a função por $W(\tilde{n}, \beta)=W(n, \bar{n}, \beta)$

Pela definição de produto de convolução notamos que

$$
W\left(n_{D_{0}}, \bar{n}_{D_{0}}, \beta\right)=\frac{e^{-\beta\left(\left(2^{(d-2)}-1\right)\left(\begin{array}{c}
\tilde{n}_{D_{0}} \\
2
\end{array}\right)\right)}}{2^{d \cdot n_{D_{0}}}}\left(W\left(\cdot, \bar{n}_{D_{1}}, 2^{(d-2)} \beta\right) * \ldots * W\left(\cdot, \bar{n}_{D_{2^{d}}}, 2^{(d-2)} \beta\right)\right)\left(n_{D_{0}}\right),
$$

onde $D_{1}, \ldots, D_{2^{d}} \in \mathcal{D}_{1}$.

Essa é a equação de grupo de renormalização que estudamos no trabalho. Uma pequena variação feita daqui para frente é considerar as duas variáveis $n$ e $\bar{n}$ ao invés de $\tilde{n}$, já que isso deixa algumas passagens mais claras. 


\section{Capítulo 4}

\section{Ponto Fixo}

A análise do ponto fixo do GR é muito importante para o estudo de sua dinâmica. Com isso queremos dizer, quando olhamos a equação que leva a função $W$ de uma hierarquia à seguinte, que formas de $W$ fazem o sistema ser autosimilar? Que tipo de função $W$ tem a mesma forma entre hierarquias do sistema? E sabendo isso gostaríamos de responder se um ponto fixo, nesse sentido, é estável ou não, ou seja, se começarmos considerando uma distribuição qualquer de partículas em uma hierarquia e "subirmos"os níveis um número suficiente de vezes, vamos em algum momento, necessariamente, chegar na forma do ponto fixo? Se sim, esse é um ponto fixo estável. Para encontrarmos um ponto fixo realizamos duas tentativas que apresentam, cada uma delas, suas vantagens e desvantagens. As duas tentativas são baseadas em um Ansatz gaussiano.

\subsection{Ansatz}

Vamos usar o seguinte Ansatz para encontrar o ponto fixo para $n$ grande:

$$
W(n, \bar{n}, \beta)=G(n, \bar{n}, \beta) V(n, \beta)
$$

onde $\bar{n}$ é a quantidade de partículas negativas do background no hipercubo em questão, $G(n, \bar{n}, \beta):=$ $e^{-r(\beta)(n-\bar{n})^{2}+b(\beta)(n-\bar{n})}$ e $V(n, \beta)$ é uma função periódica em $n$, cujo período veremos ser $k$. No futuro, muitas vezes o termo linear com $b$ será omitido, pois ele não se altera nas convoluções e, portanto, seu estudo não é tão interessante. Em um primeiro momento vamos também considerar o termo oscilatório como sendo $V(n, \beta)=1$, pare entendermos a função do termo Gaussiano na convolução. Assumimos também que $r(\beta) \propto \beta$.

Para esse Ansatz, vamos avaliar como a convolução se comporta para que possamos encontrar o ponto fixo. Simplificamos a notação definindo $2^{d}:=k$ e $2^{(d-2)} \beta:=\beta^{\prime}$, assim o objetivo agora é encontrar uma expressão para 


$$
\left(G\left(\cdot, \bar{n}_{D_{1}}, \beta^{\prime}\right) * G\left(\cdot, \bar{n}_{D_{2}}, \beta^{\prime}\right) * \cdots * G\left(\cdot, \bar{n}_{D_{k}}, \beta^{\prime}\right)\right)(n),
$$

$k$ convoluções de $G$.

Essa expressão é uma soma da forma:

$$
\sum_{\substack{n_{D_{1}}, \ldots, n_{D_{k}} \\ \sum_{j} n_{D_{j}}=n}}\left(G\left(n_{D_{1}}, \bar{n}_{D_{1}}, \beta^{\prime}\right) G\left(n_{D_{2}}, \bar{n}_{D_{2}}, \beta^{\prime}\right) \ldots G\left(n_{D_{k}}, \bar{n}_{D_{k}}, \beta^{\prime}\right)\right)(n)
$$

Vamos encontrar uma forma mais conveniente de escrever a convolução, considerando que em uma escala $(l+1)$ o background é $\bar{n}^{(l+1)}=k\left[\frac{\bar{n}^{(l)}}{k}\right]+\alpha$, onde o dígito $0 \leq \alpha<k$.

$$
\sum_{\substack{n_{D_{1}}, \ldots, n_{D_{k}} \\ \sum_{j} n_{D_{j}}=n}} \exp \left[-\sum_{i=1}^{\alpha} r\left(\beta^{\prime}\right)\left(n_{i}-\left[\frac{\bar{n}}{k}\right]-1\right)^{2}-\sum_{i=\alpha+1}^{k} r\left(\beta^{\prime}\right)\left(n_{i}-\left[\frac{\bar{n}}{k}\right]\right)^{2}\right]
$$

a parte linear que mulitplica $b$ mantém sua forma por convoluções, então não precisamos considerá-la aqui. Juntando todos os termos do expoente das exponenciais,

$\sum_{i=1}^{\alpha} r\left(\beta^{\prime}\right)\left(n_{i}-\left[\frac{\bar{n}}{k}\right]-1\right)^{2}+\sum_{i=\alpha+1}^{k} r\left(\beta^{\prime}\right)\left(n_{i}-\left[\frac{\bar{n}}{k}\right]\right)^{2}=\sum_{i=1}^{k} r\left(\beta^{\prime}\right)\left(n_{i}-\left[\frac{\bar{n}}{k}\right]\right)^{2}-2 \sum_{i=1}^{\alpha} r\left(\beta^{\prime}\right)\left(n_{i}-\left[\frac{\bar{n}}{k}\right]\right)+r\left(\beta^{\prime}\right) \alpha$ substituindo $\left[\frac{\bar{n}}{k}\right]=\frac{\bar{n}-\alpha}{k}$

$$
\frac{r\left(\beta^{\prime}\right)}{k}(n-\bar{n})^{2}+r\left(\beta^{\prime}\right)\left(\sum_{i=1}^{k} n_{i}^{2}-\frac{n^{2}}{k}\right)-2 r\left(\beta^{\prime}\right) \sum_{i=1}^{\alpha}\left(n_{i}\right)+r\left(\beta^{\prime}\right) \alpha+\frac{2 r\left(\beta^{\prime}\right)}{k} n \alpha-\frac{r\left(\beta^{\prime}\right)}{k} \alpha^{2}
$$

Todos os termos com $\alpha$ podem ser escritos como algo da forma

$$
\sum_{i=1}^{k}\left(n_{i}-z_{i}\right)^{2}-\frac{1}{k}\left(\sum_{i=1}^{k}\left(n_{i}-z_{i}\right)\right)^{2}=\sum_{i=1}^{k} n_{i}^{2}-2 \sum_{i=1}^{k} n_{i} z_{i}+\sum_{i=1}^{k} z_{i}^{2}-\frac{1}{k}\left(\sum_{i=1}^{k} n_{i}\right)^{2}+\frac{2}{k}\left(\sum_{i=1}^{k} n_{i}\right)\left(\sum_{i=1}^{k} z_{i}\right)-\frac{1}{k}\left(\sum_{i=1}^{k} z_{i}\right)^{2}
$$

e comparando as partes da equação interessantes

$$
\begin{gathered}
\left(\sum_{i=1}^{k} n_{i}^{2}-\frac{n^{2}}{k}\right)-2 \sum_{i=1}^{\alpha}\left(n_{i}\right)+\alpha+\frac{2}{k} n \alpha-\frac{1}{k} \alpha^{2} \\
=\sum_{i=1}^{k} n_{i}^{2}-2 \sum_{i=1}^{k} n_{i} z_{i}+\sum_{i=1}^{k} z_{i}^{2}-\frac{1}{k}\left(\sum_{i=1}^{k} n_{i}\right)^{2}+\frac{2}{k}\left(\sum_{i=1}^{k} n_{i}\right)\left(\sum_{i=1}^{k} z_{i}\right)-\frac{1}{k}\left(\sum_{i=1}^{k} z_{i}\right)^{2} \\
-2 \sum_{i=1}^{\alpha}\left(n_{i}\right)+\alpha+\frac{2}{k} n \alpha-\frac{1}{k} \alpha^{2}
\end{gathered}
$$




$$
=-2 \sum_{i=1}^{k} n_{i} z_{i}+\sum_{i=1}^{k} z_{i}^{2}+\frac{2}{k}\left(\sum_{i=1}^{k} n_{i}\right)\left(\sum_{i=1}^{k} z_{i}\right)-\frac{1}{k}\left(\sum_{i=1}^{k} z_{i}\right)^{2}
$$

Uma escolha clara que pode ser feita é todos o $z_{i}$ serem tais que $\sum_{i=1}^{k} z_{i}=\alpha$. Dessa forma resta

$$
-2 \sum_{i=1}^{\alpha}\left(n_{i}\right)+\alpha=-2 \sum_{i=1}^{k} n_{i} z_{i}+\sum_{i=1}^{k} z_{i}^{2}
$$

a escolha que faz com que essa última equação seja válida é

$$
z_{i}= \begin{cases}1 & 1 \leq i \leq \alpha \\ 0 & \alpha<i \leq k\end{cases}
$$

Assim podemos escrever a parte exponencial da gaussiana como

$$
\frac{r\left(\beta^{\prime}\right)}{k}(n-\bar{n})^{2}+r\left(\beta^{\prime}\right)\left(\sum_{i=1}^{k}\left(n_{i}-z_{i}\right)^{2}-\frac{1}{k}(n-\alpha)^{2}\right)
$$

e

$$
\sum_{\substack{n_{D_{1}}, \ldots, n_{D_{k}} \\ \sum_{j} n_{D_{j}}=n}} \exp \left[-\frac{r\left(\beta^{\prime}\right)}{k}(n-\bar{n})^{2}-r\left(\beta^{\prime}\right)\left(\sum_{i=1}^{k}\left(n_{i}-z_{i}\right)^{2}-\frac{1}{k}(n-\alpha)^{2}\right)\right] .
$$

Podemos ainda realizar uma mudança de variáveis, para simplificar as equações $n_{i}^{\prime}=n_{i}-z_{i}$ e $n^{\prime}=$ $n-\alpha$.

$$
e^{-\frac{r\left(\beta^{\prime}\right)}{k}(n-\bar{n})^{2}} \sum_{\substack{n_{D_{1}}^{\prime}, \ldots, n_{D_{k}}^{\prime} \\ \sum_{j} n_{D_{j}}^{\prime}=n^{\prime}}} \exp \left[-r\left(\beta^{\prime}\right)\left(\sum_{i=1}^{k} n_{i}^{\prime 2}-\frac{1}{k} n^{\prime 2}\right)\right]
$$

Uma das formas de calcular a soma com o vinculo é iterativamente, considerando

$$
\sum_{m_{1}=-1}^{n^{\prime}}\left(G\left(n_{D_{1}}, \frac{\bar{n}}{k}, \beta^{\prime}\right) * \cdots * G\left(n_{D_{k-1}}, \frac{\bar{n}}{k}, \beta^{\prime}\right)\right)\left(m_{1}\right) G\left(\left(n-m_{1}\right), \frac{\bar{n}}{k}, \beta^{\prime}\right) .
$$

Pela mesma lógica, no $k$-ésimo passo

$$
e^{r\left(\beta^{\prime}\right) \frac{n^{2}}{k}} \sum_{m_{1}=-1}^{n^{\prime}} \ldots \sum_{m_{\alpha}=-1}^{m_{\alpha-1}} \sum_{m_{\alpha+1}=0}^{m_{\alpha}} \ldots \sum_{m_{k-1}=0}^{m_{k-2}} G\left(m_{k-1}, \frac{\bar{n}}{k}, \beta^{\prime}\right) \ldots G\left(\left(m_{1}-m_{2}\right), \frac{\bar{n}}{k}, \beta^{\prime}\right) G\left(\left(n-m_{1}\right), \frac{\bar{n}}{k}, \beta^{\prime}\right) .
$$

Com o ansatz temos que, completando quadrados no expoente, podemos separar o termo gaussiano que depende do background e obtemos

$$
=e^{-\frac{r\left(\beta^{\prime}\right)}{k}(n-\bar{n})^{2}} \sum_{m_{1}=-1}^{n^{\prime}} \cdots \sum_{m_{\alpha}=-1}^{m_{\alpha-1}} \sum_{m_{\alpha+1}=0}^{m_{\alpha}} \cdots \sum_{m_{k-1}=0}^{m_{k-2}} \exp \left[-r\left(\beta^{\prime}\right)\left(\left(n^{\prime}-m_{1}\right)^{2}+\sum_{i=2}^{k-1}\left(m_{i-1}-m_{i}\right)^{2}+m_{k-1}^{2}-\frac{1}{k} n^{\prime 2}\right)\right] .
$$

Essa expressão quase nos dá um ponto fixo, mas temos fator convolutivo que é difícil de analisar. Vamos olhar numéricamente um exemplo para $r=1$ e $k=1$ (3 dimensões) sem o background de 
como fica o termo da soma, ou seja, sem considerar $e^{-\frac{r\left(\beta^{\prime}\right)}{k}(n-\bar{n})^{2}}$, em função de $n$.
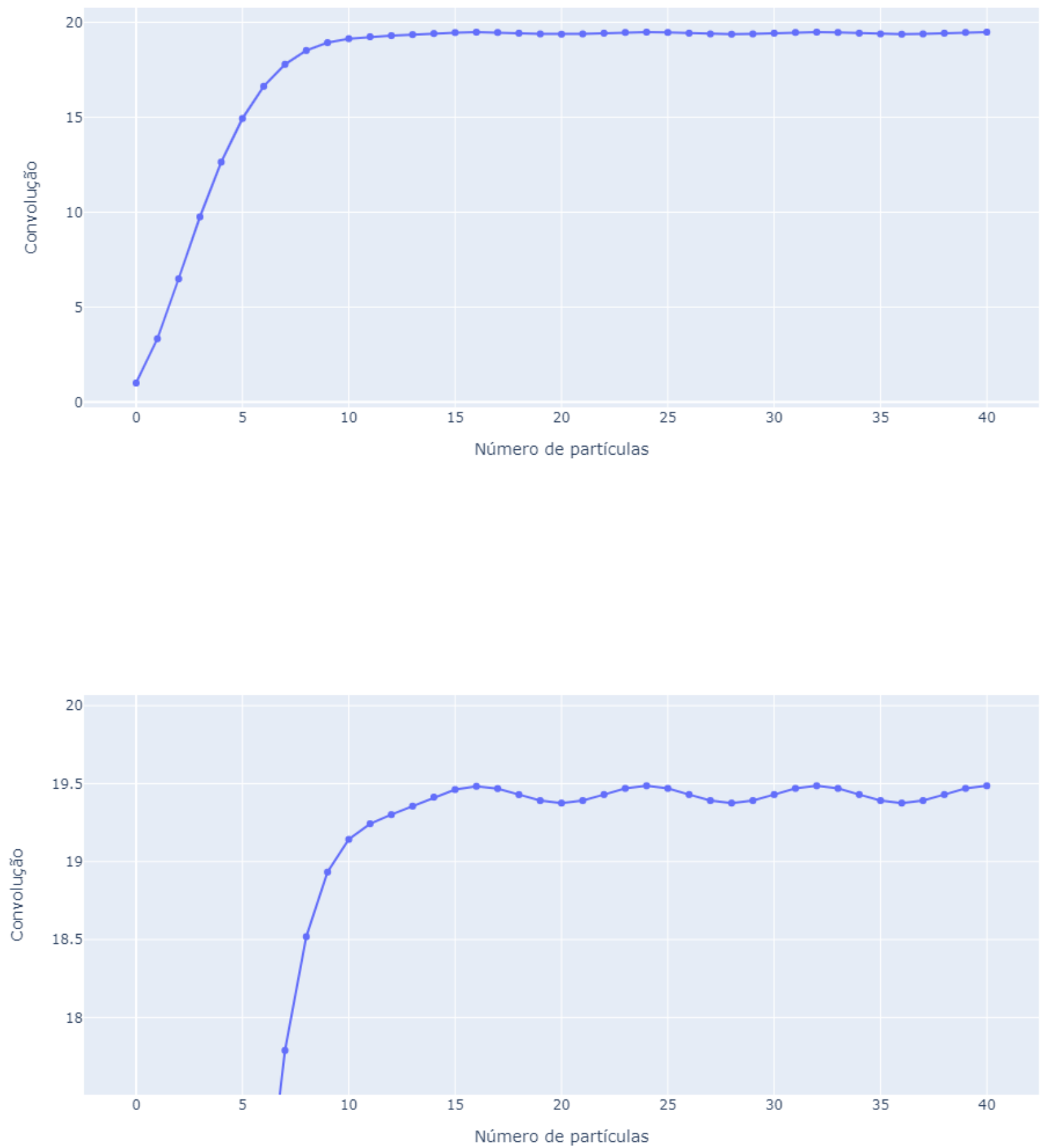

Notamos que há um carater oscilatório nessa convolução e veremos que essas oscilações se dão em torno de um valor fixo para $n$ grande. Podemos ainda analisar o efeito que uma escolha de background tem sobre essas oscilações $k$-periódicas, ou seja, diferentes valores de $\alpha$ 

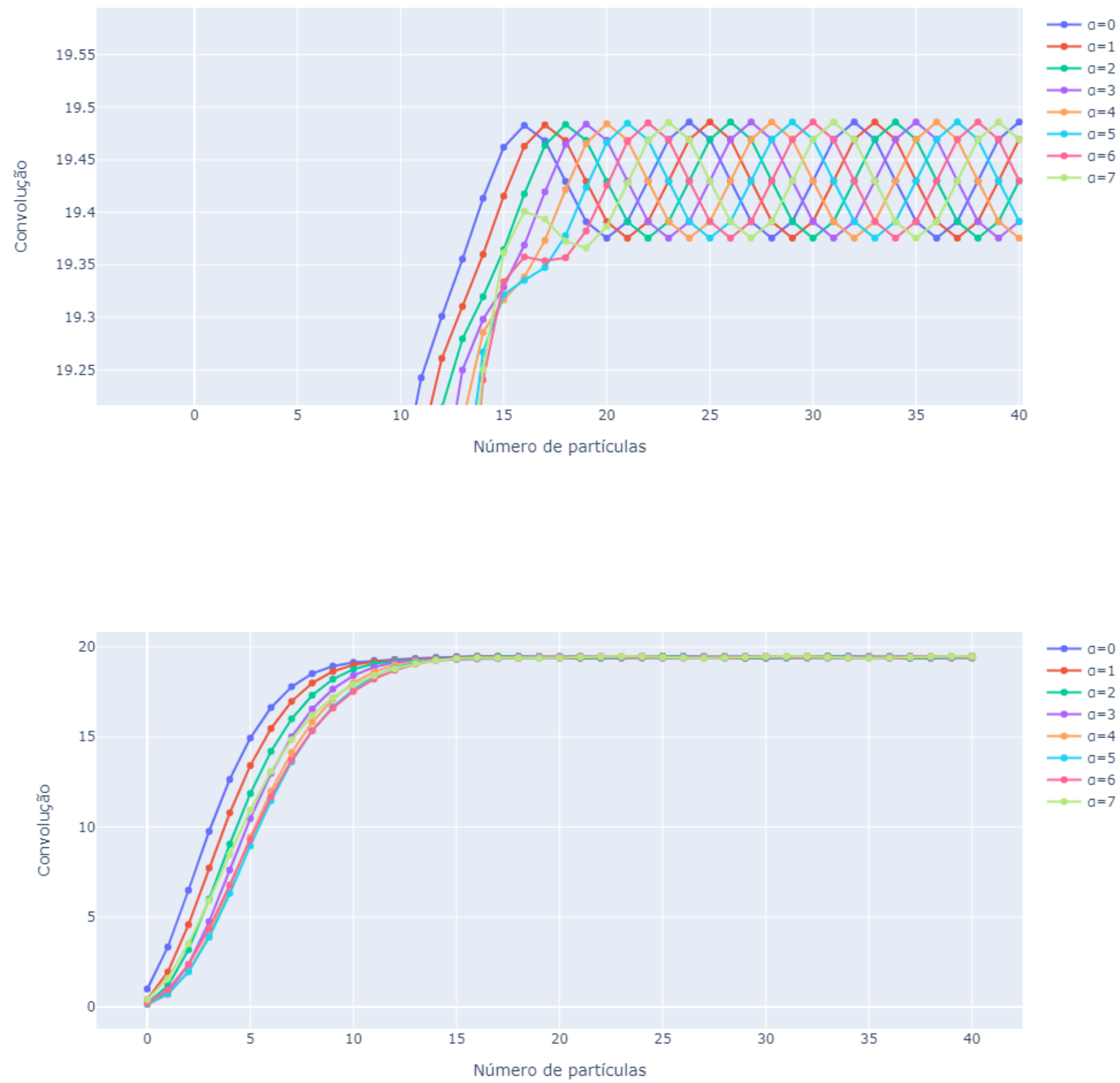

Essa forma (4.14) é muito mais conveniente, ela permite que os termos da gaussiana sejam manipulados de forma mais simples. Ela também nos permite plotar gráficos exatos para a convolução e comparar com a soma de Poisson nas seções futuras. É essa também a equação que nos permite ver que valores diferentes de $\alpha$ correspondem apenas a um deslocamento das oscilações para grandes valores de $n$. Vamos assim, por simplicidade, assumir que o background é um múltiplo de $k$ na escala menor, para fazermos as contas $\operatorname{com} \alpha=0$.

Por fim observamos que ainda há uma fase transiente na qual a convolução não é oscilatória, mas ainda se aproxima desse regime. Uma pequena discussão sobre essa fase será feita mais adiante. 


\subsection{A Convolução Contínua}

Um primeiro impulso natural e ingênuo para tratar esse sistema pode ser aproximar as somas das convoluções discretas, que são complicadas, por convoluções contínuas. Vamos estudar essa ideia sem nos preocuparmos rigorosamente com o erro, mas imaginamos que ele não deva ser grande no limite termodinâmico. Essa abordagem começa a se mostrar relevante, quando notamos que o valor da convolução contínua é $\sqrt{\frac{\pi^{k-1}}{r^{k-1} k}}$. Podemos verificar numéricamente que esse é o valor em torno do qual a convolução discreta oscila e na seção sobre a fórmula de Poisson mostraremos analiticamente que esse é o caso. Dessa forma, não só para a versão da convolução contínua, mas também para a discreta, temos que o fator $\sqrt{\frac{\pi^{k-1}}{r^{k-1} k}}$ é uma normalização conveniente nas iterações da equação de grupo de renormalização.

$k$ convoluções contínuas do ponto fixo nos dão

$$
\sqrt{\frac{\pi^{k-1}}{k\left(r\left(\beta^{\prime}\right)\right)^{k-1}}} e^{-\frac{r\left(\beta^{\prime}\right)}{k}\left(n-k \frac{\bar{n}}{2^{d}}\right)^{2}+b\left(\beta^{\prime}\right)\left(n-k \frac{\bar{n}}{2^{d}}\right)} .
$$

Dividindo por uma normalização conveniente nos resta

$$
e^{-\frac{r\left(\beta^{\prime}\right)}{2^{d}}(n-\bar{n})^{2}+b\left(\beta^{\prime}\right)(n-\bar{n})} .
$$

O Ansatz aplicado na equação de GR resulta em:

$$
e^{-r(\beta)(n-\bar{n})^{2}+b(\beta)(n-\bar{n})}=\frac{e^{-\beta\left(\left(2^{(d-2)}-1\right)\left(\begin{array}{c}
n-\bar{n} \\
2
\end{array}\right)\right)}}{2^{d(n-\bar{n})}} e^{-\frac{r\left(\beta^{\prime}\right)}{2^{d}}(n-\bar{n})^{2}+b\left(\beta^{\prime}\right)(n-\bar{n})} .
$$

Podemos então encontrar uma forma para as funções $b(\beta)$ e $r(\beta)$. Vamos reescrever (4.17) da seguinte forma

$$
\begin{gathered}
-r(\beta)(n-\bar{n})^{2}+b(\beta)(n-\bar{n}) \\
=-\beta\left(\left(2^{(d-2)}-1\right)\left(\begin{array}{c}
(n-\bar{n})_{D_{0}} \\
2
\end{array}\right)\right)-d(n-\bar{n}) \log (2)-\frac{r\left(\beta^{\prime}\right)}{2^{d}}(n-\bar{n})^{2}+b\left(\beta^{\prime}\right)(n-\bar{n}) .
\end{gathered}
$$

Notamos aqui que, $r$ só multiplica dependências em $(n-\bar{n})^{2}$ e $b$ só multiplica dependências em $(n-\bar{n})$. Dessa forma vamos considerar o sistema dos termos que multiplicam cada um desses fatores, $(n-\bar{n})^{2}$ e $(n-\bar{n})$. Vamos evidenciar de qual hierarquia, $l$ ou $l+1$, vem os parâmetros, para nos ajudar a percereber que o mapa a seguir converge independente do valor de $\beta$ para um ponto fixo, se assumirmos que $r(\beta) \propto \beta$.

$$
\left\{\begin{array}{l}
r(\beta)=\frac{\beta\left(2^{(d-2)}-1\right)}{2}+\frac{r\left(2^{d-2} \beta\right)}{2^{d}} \\
b(\beta)=\frac{\beta\left(2^{(d-2)}-1\right)}{2}-d \log (2)+b\left(2^{d-2} \beta\right)
\end{array}\right.
$$

Vamos primeiro resolver a equação para $r$. Podemos substituir iterativamente os valores de 
$r\left(2^{(d-2)} \beta\right), r\left(2^{2(d-2)} \beta\right), \ldots$

$$
\begin{gathered}
r(\beta)=\frac{\beta\left(2^{d-2}-1\right)}{2}\left[1+\frac{2^{d-2}}{2^{d}}+\frac{2^{2(d-2)}}{2^{2 d}}+\frac{2^{3(d-2)}}{2^{3 d}}+\ldots\right]=\frac{\beta\left(2^{d-2}-1\right) 2^{d-2}}{2} \sum_{i=0}^{\infty} 2^{-2 i} \\
r(\beta)=2 \frac{\beta\left(2^{d-2}-1\right)}{3}
\end{gathered}
$$

Agora resolvemos a equação para $b$ :

$$
\begin{gathered}
b(\beta)=\frac{\beta\left(2^{d-2}-1\right)}{2} \sum_{i=0}^{k-1} 2^{i(d-2)}-k \log (2)+b\left(2^{k(d-2)} \beta\right) \\
b(\beta)=\frac{\beta}{2}\left(2^{k(d-2)}-1\right)-k \log (2)+b\left(2^{k(d-2)} \beta\right)
\end{gathered}
$$

O que fazemos agora é renomear $2^{k(d-2)} \beta=: \beta^{\prime}$, portanto $2^{k}=\left(\frac{\beta^{\prime}}{\beta}\right)^{\frac{1}{d-2}}$ e assim

$$
\begin{gathered}
b(\beta)=\frac{1}{2}\left(\beta^{\prime}-\beta\right)-\frac{d}{d-2} \log \left(\frac{\beta^{\prime}}{\beta}\right)+b\left(\beta^{\prime}\right) \\
{\left[b(\beta)+\frac{1}{2} \beta-\frac{d}{d-2} \log (\beta)\right]-\left[b\left(\beta^{\prime}\right)+\frac{1}{2} \beta^{\prime}-\frac{d}{d-2} \log \left(\beta^{\prime}\right)\right]=0} \\
\int_{\beta}^{\beta^{\prime}} b^{\prime}(x)+\frac{1}{2}-\frac{d}{d-2} \frac{1}{x} d x=0
\end{gathered}
$$

Como isso tem de valer para todas as combinações de $\beta$ e $\beta^{\prime}$, então uma solução é:

$$
b^{\prime}(x)+\frac{1}{2}-\frac{d}{d-2} \frac{1}{x}=0
$$

e assim

$$
b(x)=\frac{1}{2} x-\frac{d}{d-2} \log x+c
$$

Essa constante não deve importar, pois grandezas independentes de $\beta$ vão acabar sendo incluidas na constante de normalização que já definimos.

Juntanto todos esses resultados, chegamos finalmente a uma forma para o ponto fixo.

$$
W^{*}(n, \bar{n}, \beta)=e^{-2 \frac{\beta\left(2^{d-2}-1\right)}{3}(n-\bar{n})^{2}+\left(\frac{1}{2} \beta-\frac{d}{d-2} \log \beta\right)(n-\bar{n})}
$$

O problema com essa abordagem é que não temos uma estimativa precisa do erro cometido com a aproximação da convolução discreta pela contínua, mas é um indício de que essa forma de solução deve apresentar resultados. 


\subsection{Maior Contribuição da Soma}

Para tratar (4.13) vamos primeiro entender quais são os máximos das gaussianas que aparecem, na expectativa de que as contribuições sejam muito pequenas longe deles. Para enxergarmos isso, vamos escrever a soma $M_{n}$ de uma forma mais conveniente:

$$
\begin{aligned}
M_{n} & =\sum_{m_{1}=-1}^{n^{\prime}} \ldots \sum_{m_{\alpha}=-1}^{m_{\alpha-1}} \sum_{m_{\alpha+1}=0}^{m_{\alpha}} \cdots \sum_{m_{k-1}=0}^{m_{k-2}} e^{-r\left(\beta^{\prime}\right)\left(\left(n^{\prime}-m_{1}\right)^{2}+\sum_{i=1}^{k-2}\left(m_{i}-m_{i+1}\right)^{2}+m_{k-1}^{2}-\frac{1}{k} n^{\prime 2}\right)} \\
& =\sum_{m_{1}=-1}^{n^{\prime}} \cdots \sum_{m_{\alpha}=-1}^{m_{\alpha-1}} \sum_{m_{\alpha+1}=0}^{m_{\alpha}} \cdots \sum_{m_{k-1}=0}^{m_{k-2}} e^{-r\left(\beta^{\prime}\right)\left((\tilde{m}, y)+(y, \tilde{m})+\left(\tilde{m}, \tilde{J}_{k} \tilde{m}\right)+n^{\prime 2}\left(1-\frac{1}{k}\right)\right)}
\end{aligned}
$$

onde

$$
\begin{aligned}
& \tilde{m}:=\left(\begin{array}{c}
m_{1} \\
\vdots \\
m_{k-1}
\end{array}\right) \\
& \tilde{J}_{k}:=\left(\begin{array}{ccccccc}
2 & -1 & 0 & & & & \\
-1 & 2 & -1 & & & 0 & \\
0 & -1 & 2 & & & & \\
& & & \ddots & & & \\
& & & & 2 & -1 & 0 \\
& 0 & & & -1 & 2 & -1 \\
& & & & 0 & -1 & 2
\end{array}\right) \\
& y:=\left(\begin{array}{c}
-n^{\prime} \\
0 \\
\vdots \\
0
\end{array}\right)
\end{aligned}
$$

Notamos de (4.31) que o vetor $\tilde{m}^{*}$ que maximiza o somando é o que satisfaz o sistema de equações

$$
\left\{\begin{array}{l}
2 m_{1}^{*}-m_{2}^{*}-n^{\prime}=0 \\
-m_{1}^{*}+2 m_{2}-^{*} m_{3}^{*}=0 \\
\vdots \\
-m_{i-1}^{*}+2 m_{i}^{*}-m_{i+1}^{*}=0 \\
\vdots \\
-m_{k-3}^{*}+2 m_{k-2}^{*}-m_{k-1}^{*}=0 \\
-m_{k-2}^{*}+2 m_{k-1}^{*}=0
\end{array}\right.
$$


o segredo para resolver esse sistema facilmente é começar de baixo para cima, que dá as relações:

$$
\begin{gathered}
m_{k-1}^{*}=\frac{1}{k} n^{\prime} \\
m_{k-2}^{*}=\frac{2}{k} n^{\prime} \\
\vdots \\
m_{i}^{*}=\frac{k-i}{k} n^{\prime} \\
\vdots \\
m_{1}^{*}=\frac{k-1}{k} n^{\prime}
\end{gathered}
$$

\subsection{Limites de M}

Proposição 2. Para $C_{1}, c_{2}>0$ (ou $\left.D_{1}, d_{2}>0\right)$ vale

$$
\begin{gathered}
\left|M_{n}-M_{n}^{\varepsilon}\right| \leq C_{1} e^{-c_{2} n^{2}} \\
\text { ou } \left.\left|\tilde{M}-M_{n}^{\varepsilon}\right| \leq D_{1} e^{-d_{2} n^{2}}\right) .
\end{gathered}
$$

Tendo os máximos, queremos mostrar agora que as contribuições principais da somatória (4.13) se darão em torno deles, com isso a ideia é considerar

$$
\tilde{M}_{n}:=\sum_{m_{1}=-\infty}^{\infty} \sum_{m_{2}=-\infty}^{\infty} \ldots \sum_{m_{k-1}=-\infty}^{\infty} e^{-r\left(\beta^{\prime}\right)\left(\left(n^{\prime}-m_{1}\right)^{2}+\sum_{i=1}^{k-2}\left(m_{i}-m_{i+1}\right)^{2}+m_{k-1}^{2}-\frac{n^{\prime 2}}{k}\right)}
$$

no lugar de

$$
M_{n}=\sum_{m_{1}=-1}^{n^{\prime}} \ldots \sum_{m_{\alpha}=-1}^{m_{\alpha-1}} \sum_{m_{\alpha+1}=0}^{m_{\alpha}} \ldots \sum_{m_{k-1}=0}^{m_{k-2}} e^{-r\left(\beta^{\prime}\right)\left(\left(n^{\prime}-m_{1}\right)^{2}+\sum_{i=1}^{k-2}\left(m_{i}-m_{i+1}\right)^{2}+m_{k-1}^{2}-\frac{n^{\prime 2}}{k}\right)} .
$$

Para isso definimos a função

$$
\begin{gathered}
f\left(n^{\prime}, m_{1}, \ldots, m_{k-1}\right):=e^{-r\left(\beta^{\prime}\right)\left(\left(n^{\prime}-m_{1}\right)^{2}+\sum_{i=1}^{k-2}\left(m_{i}-m_{i+1}\right)^{2}+m_{k-1}^{2}-\frac{n^{\prime 2}}{k}\right)} . \\
g\left(n^{\prime}, m_{i}, \ldots, m_{k-1}\right):=\left(n^{\prime}-m_{1}\right)^{2}+\sum_{i=1}^{k-2}\left(m_{i}-m_{i+1}\right)^{2}+m_{k-1}^{2}-\frac{1}{k} n^{\prime 2}
\end{gathered}
$$

Notamos que o maior valor dessa função é o que minimiza $g$

$$
\begin{gathered}
g\left(n^{\prime}, m_{i}, \ldots, m_{k-1}\right)=(\tilde{m}, y)+(y, \tilde{m})+\left(\tilde{m}, \tilde{J}_{k} \tilde{m}\right)+n^{\prime 2}\left(1-\frac{1}{k}\right) \\
f\left(n^{\prime}, m_{1}^{*}, \ldots, m_{k-1}^{*}\right)=1 .
\end{gathered}
$$

Se qualquer $m_{i}$ desviar desse valor máximo, como por exemplo $m_{i}=m_{i}^{*}+\delta$, termos que, o termo 
que maximiza a função $f$, sujeita a esse vínculo é

$$
\begin{aligned}
& m_{k-1}=m_{k-1}^{*}+\frac{1}{k-i} \delta \\
& \vdots \\
& m_{i+1}=m_{i+1}^{*}+\frac{k-i-1}{k-i} \delta \\
& m_{i}=m_{i}^{*}+\delta \\
& m_{i-1}=\frac{i-1}{i}\left(m_{i}^{*}+\delta\right)+\frac{1}{i} n^{\prime} \\
& \vdots \\
& m_{i-j}=\frac{i-j}{i}\left(m_{i}^{*}+\delta\right)+\frac{j+1}{i} n^{\prime} \\
& \vdots \\
& m_{1}=\frac{1}{i}\left(m_{i}^{*}+\delta\right)+\frac{i-1}{i} n^{\prime}
\end{aligned}
$$

e assim

$$
\begin{gathered}
m_{k-1}^{2}+\left(m_{k-2}-m_{k-1}\right)^{2}+\left(m_{k-3}-m_{k-2}\right)^{2}+\cdots+\left(m_{i}-m_{i+1}\right)^{2}+\left(m_{i-1}-m_{i}\right)^{2}+\left(m_{i-2}-m_{i-1}\right)^{2}+\ldots \\
+\left(m_{1}-m_{2}\right)^{2}+\left(n^{\prime}-m_{1}\right)^{2} \\
=\left(m_{k-1}^{*}+\frac{1}{k-i} \delta\right)^{2}+\left(m_{k-2}^{*}-m_{k-1}^{*}+\frac{1}{k-i} \delta\right)^{2}+\left(m_{k-3}^{*}-m_{k-2}^{*}+\frac{1}{k-i} \delta\right)^{2}+\cdots+\left(m_{i}^{*}-m_{i+1}^{*}+\frac{1}{k-i} \delta\right)^{2} \\
+\left(\frac{1}{i}\left(m_{i}^{*}+\delta\right)-\frac{1}{i} n^{\prime}\right)^{2}+\left(\frac{1}{i}\left(m_{i}^{*}+\delta\right)-\frac{1}{i} n^{\prime}\right)^{2}+\cdots+\left(\frac{1}{i}\left(m_{i}^{*}+\delta\right)-\frac{1}{i} n^{\prime}\right)^{2}+\left(\frac{1}{i}\left(m_{i}^{*}+\delta\right)-\frac{1}{i} n^{\prime}\right)^{2} \\
=\left(m_{k-1}^{*}\right)^{2}+\left(m_{k-2}^{*}-m_{k-1}^{*}\right)^{2}+\left(m_{k-3}^{*}-m_{k-2}^{*}\right)^{2}+\cdots+\left(m_{i}^{*}-m_{i+1}^{*}\right)^{2}+\frac{1}{k-i} \delta^{2}+2 \frac{1}{k-i} \delta m_{i}^{*}+\frac{1}{i}\left(m_{i}^{*}+\delta-n^{\prime}\right)^{2} \\
=\left(\frac{n^{\prime}}{k}\right)^{2}(k-i)+\frac{1}{k-i} \delta^{2}+2 \frac{1}{k} \delta n^{\prime}+\frac{1}{i}\left(\delta-\frac{i}{k} n^{\prime}\right)^{2} \\
=\frac{n^{\prime 2}}{k}+\left(\frac{1}{k-i}+\frac{1}{i}\right) \delta^{2}
\end{gathered}
$$

Com isso percebemos que

$$
f\left(n^{\prime}, m_{1}, \ldots, m_{i}^{*}+\delta, \ldots, m_{k-1}\right) \leq e^{-r\left(\frac{1}{k-i}+\frac{1}{i}\right) \delta^{2}}
$$

e concluímos que o erro de se somar ao máximo um termo que difere de $\delta$ em uma componente do valor máximo da função é $\mathcal{O}\left(\exp \left(-r\left(\frac{1}{k-i}+\frac{1}{i}\right) \delta^{2}\right)\right.$. Ou seja, termos longe do centro tem contribuição muito pequena para a somatória e, portanto, podemos considerar $\tilde{M}_{n}$ no lugar de $M_{n}$ cometendo um erro $\mathcal{O}\left(\exp \left(-r\left(\frac{1}{k-i}+\frac{1}{i}\right) \delta^{2}\right)\right)$. Definimos o conjunto $A_{\varepsilon}=\bigcup_{i=1}^{k-1}\left|m_{i}-m_{i}^{*}\right| \leq \varepsilon n$ e seu complementar $A_{\varepsilon}^{c}$

Se considerarmos $\delta=n^{\prime} \varepsilon$ e definirmos $M_{n}^{\varepsilon}$ como sendo

$$
M_{n}^{\varepsilon}:=\sum_{m_{1}, \ldots, m_{k-1} \in A_{\varepsilon}} f\left(n^{\prime}, m_{1}, \ldots, m_{k-1}\right)
$$


as somas de $f\left(n^{\prime}, m_{1}, \ldots, m_{k-1}\right)$ em $m_{1}, \ldots, m_{k-1}$, para todos os termos em que os $m_{i}$ estejam dentro de $\left(m_{i}^{*}-\varepsilon n^{\prime}, m_{i}^{*}+\varepsilon n^{\prime}\right)$, que é o intervalo centrado em torno de sua contribuição máxima, temos que, a maior contribuição de $\left|M_{n}-M_{n}^{\varepsilon}\right|$ será o termo $R$ em que todos os $m_{j} \in A_{\varepsilon}$ exceto um $m_{i} \in A_{\varepsilon}^{c}$

$$
R \leq 2 \sum_{\delta=n^{\prime} \varepsilon}^{\infty} e^{-r\left(\frac{1}{k-i}+\frac{1}{i}\right) \delta^{2}}\left(2 \varepsilon n^{\prime}\right)^{k-2}
$$

Essa não é uma prova completa, mas uma argumentação convincente que as constantes da proposição devem existir.

\subsection{A Fórmula de Poisson}

A fórmula de Poisson nos permite escrever a soma de uma função como sendo, no espaço de Fourier, a transformada da própria função e a soma toda em uma exponencial complexa. A forma dessa soma é:

$$
\sum_{m \in \mathbb{Z}^{k-1}} f(x+L m)=\frac{1}{(2 L)^{n}} \sum_{k \in \mathbb{Z}^{k-1}} \hat{f}\left(\frac{k}{2 L}\right) e^{2 \pi i \frac{k}{2 L} \cdot x} .
$$

A prova dessa igualdade pode ser encontrada em [5] e nos apêndices ao final do texto.

Agora conectamos isso com o nosso produto de convolução. Notamos que $\tilde{M}_{n}$ é justamente uma função na forma de uma soma de Poisson.

No nosso caso usamos a forma multidimensional análoga

$$
\sum_{m \in \mathbb{Z}^{k-1}} f(x+m)=\sum_{\xi \in \mathbb{Z}^{k-1}} \hat{f}(\xi) e^{2 \pi i \xi \cdot x}
$$

onde

$$
\hat{f}(\xi)=\int_{\mathbb{R}^{k-1}} \cdots \int_{f} f(x) e^{-2 \pi i \xi \cdot x} d^{k-1} x,
$$

para uma função $f: \mathbb{R}^{k-1} \rightarrow \mathbb{R}$ que seja de quadrado integrável.

Observamos que, pela análise feita anteriormente, podemos extender os limites da soma $M_{n}$ para infinito cometendo um erro gaussiano e assim podemos usar a fórmula de Poisson. Mais informações e referências podem ser encontradas no apêndice.

$$
\tilde{M}_{n}=\sum_{m_{1}=-\infty}^{\infty} \ldots \sum_{m_{k-1}=-\infty}^{\infty} f(\mathfrak{x}+\tilde{m})
$$

Vamos usar a forma mais conveniente de $f$ :

$$
\sum_{\substack{n_{D_{1}}, \ldots, n_{D_{k}} \\ \sum_{j} n_{D_{j}}=n}} \exp \left[-r\left(\beta^{\prime}\right)\left(\sum_{i=1}^{k}\left(n_{i}-z_{i}\right)^{2}-\frac{1}{k}(n-\alpha)^{2}\right)\right]
$$


Para estudar essas oscilações vamos utilizar a fórmula de Poisson e para isso precisamos de $M_{n}$ na forma 4.31

$$
\begin{gathered}
M_{n}=\sum_{m_{1}=-1}^{n^{\prime}} \ldots \sum_{m_{\alpha}=-1}^{m_{\alpha-1}} \sum_{m_{\alpha+1}=0}^{m_{\alpha}} \cdots \sum_{m_{k-1}=0}^{m_{k-2}} e^{-r\left(\beta^{\prime}\right)\left((\tilde{m}, \mathrm{y})+(y, \tilde{m})+\left(\tilde{m}, \tilde{J}_{k} \tilde{m}\right)+n^{\prime 2}\left(1-\frac{1}{k}\right)\right)} \\
M_{n}=\sum_{m_{1}=0}^{n} \sum_{m_{2}=0}^{m_{1}} \cdots \sum_{m_{k-1}=0}^{m_{k-2}} e^{-r\left(\beta^{\prime}\right)\left(\tilde{m}+\tilde{J}_{k}^{-1} y, \tilde{J}_{k}\left(\tilde{m}+\tilde{J}_{k}^{-1} y\right)\right)}
\end{gathered}
$$

denotamos agora

$$
x:=\tilde{J}_{k}^{-1} y
$$

e assim denotamos a função

$$
f(\mathfrak{x}+\tilde{m}):=e^{-r\left(\tilde{m}+x, \tilde{J}_{k}(\tilde{m}+x)\right)} .
$$

Com todas essas considerações, vamos escrever, novamente, o mapa do GR extendendo as somas, para $n$ grande. Os termos com somente $y$ e $n$ são constantes que não são integradas, dessa forma vamos considerar $f$ sem esses termos.

$$
\tilde{M}_{n}=\sum_{m \in \mathbb{Z}^{k-1}} e^{-r\left(\beta^{\prime}\right)\left(m+x, \tilde{J}_{k-1}(m+x)\right)}
$$

Para utilizarmos a fórmula de Poisson precisamos primeiro calcular

$$
\hat{f}(\xi)=\int_{\mathbb{R}^{k-1}} \cdots \int^{-r\left(\beta^{\prime}\right)\left(x+i \frac{\pi}{r} \tilde{J}_{k}^{-1} \xi, \tilde{J}_{k}\left(x+i \frac{\pi}{r} \tilde{J}_{k}^{-1} \xi\right)\right)-\frac{\pi^{2}}{r}\left(\xi, \tilde{J}_{k}^{-1} \xi\right)} d^{k-1} x=\sqrt{\frac{\left(\pi / r\left(\beta^{\prime}\right)\right)^{k-1}}{\operatorname{det} \tilde{J}_{k}}} e^{-\frac{\pi^{2}}{r}\left(\xi, \tilde{J}_{k}^{-1} \xi\right)}
$$

O determinante de $\tilde{J}_{k}$ é igual a $k$. Isso pode ser visto pela fórmula recursiva

$$
\operatorname{det} \tilde{J}_{i}=2 \operatorname{det} \tilde{J}_{i-1}-\operatorname{det} \tilde{J}_{i-2}
$$

para $4 \leq i \leq k$, det $\tilde{J}_{2}=2 \mathrm{e} \operatorname{det} \tilde{J}_{3}=3$.

Como podemos ver no corolário 4.2 de [9], a inversa dessa matriz é

$$
\left(\tilde{J}_{k}^{-1}\right)_{i j}=\left\{\begin{array}{cc}
i\left(1-\frac{j}{k}\right) & \text { se } i \leq j \\
j\left(1-\frac{i}{k}\right) & \text { se } i>j
\end{array}\right.
$$

e assim 


$$
\begin{aligned}
& (\mathfrak{x})_{l}=\left\{\begin{array}{cr}
-(n-\alpha)\left(1-\frac{l}{k}\right)-\alpha+l & \text { se } l<\alpha \\
-(n-\alpha)\left(1-\frac{l}{k}\right) & \text { se } l \geq \alpha
\end{array}\right. \\
& (\xi, \mathfrak{x})=-(n-\alpha) \sum_{j=1}^{k-1}\left(1-\frac{j}{k}\right) \xi_{j}-\sum_{j=1}^{\alpha-1}(\alpha-j) \xi_{j}
\end{aligned}
$$

O segundo termo é, com certeza, um número inteiro e, portanto, pode ser desconsiderado. Um número inteiro multiplicado por $2 \pi i$ não contribui de forma nenhuma como fase. Notamos com essa expressão que as somas correspondem a uma função de $n$ periódica, de período $k$ e que a excolha do termo $\alpha$ corresponde simplesmente a um deslocamento das oscilações de $\alpha$ unidades. Isso também pode ser observado nos exemplos numéricos da convolução exata.

Concluindo:

$$
\begin{aligned}
e^{-r(\beta)(n-\bar{n})^{2}+b(\beta)(n-\bar{n})} & =\frac{e^{-\beta\left(\left(2^{(d-2)}-1\right)\left(\begin{array}{c}
n-\bar{n} \\
2
\end{array}\right)\right)}}{k^{n} C} e^{-\frac{r\left(\beta^{\prime}\right)}{k}(n-\bar{n})^{2}+b(\beta)(n-\bar{n})} \tilde{M}_{n} \\
\tilde{M}_{n}=\sum_{m \in \mathbb{Z}^{k-1}} e^{-r\left(\beta^{\prime}\right)\left(m+x, \tilde{J}_{k-1}(m+x)\right)} & \sqrt{\frac{\left(\pi / r\left(\beta^{\prime}\right)\right)^{k-1}}{k}} \sum_{\xi \in \mathbb{Z}^{k-1}} e^{-\frac{\pi^{2}}{r}\left(\xi, \tilde{J}_{k-1}^{-1} \xi\right)} e^{-2 \pi i(n-\alpha) \sum_{j=1}^{k-1} \frac{j}{k} \xi_{j}}
\end{aligned}
$$

Essa última equação nos mostra um fenômeno interessante, que a equação de ponto fixo tem $W^{*}(n, \bar{n}, \beta, x)$ igual a um $W^{*}$ da mesma forma, com uma outra escala em $r$ e $\beta$, mas essencialmente a mesma forma, só que multiplicado por uma função

$$
V:=\sqrt{\frac{\left(\pi / r\left(\beta^{\prime}\right)\right)^{k-1}}{k}} \sum_{\xi \in \mathbb{Z}^{k-1}} e^{-\frac{\pi^{2}}{r}\left(\xi, \tilde{J}_{k-1}^{-1} \xi\right)} e^{-2 \pi i(n-\alpha) \sum_{j=1}^{k-1} \frac{j}{k} \xi_{j}}
$$

e ainda essa função, como podemos notar pelo termo da exponencial complexa na soma, é periódica de período $k$ com relação a $n$, ou seja uma Gaussiana vezes um termo que oscila. Outra coisa interessante de se notar é que o termo da raiz é justamente o que ocorre quando fazemos a convolução contínua, ou seja, esperamos que as oscilações se deem em torno do valor do contínuo.

\subsection{Caracterizando as Oscilações}

Nessa seção vamos tentar caracterizar a função periódica em $n$. Queremos definir sua amplitude de oscilação para $n$ grande e o valor em torno da qual ela oscila. Para isso basta retirar o termo zero da exponencial e assim fica claro que as oscilações são em torno do valor da convolução contínua. 


$$
\begin{gathered}
\sum_{\xi \in \mathbb{Z}^{k-1}} e^{-\frac{\pi^{2}}{r}\left(\xi, \tilde{J}_{k}^{-1} \xi\right)} e^{2 \pi i \xi \cdot x}=1+\sum_{\xi \in \mathbb{Z}^{k-1} \backslash 0} e^{-\frac{\pi^{2}}{r}\left(\xi, \tilde{J}_{k}^{-1} \xi\right)} e^{2 \pi i \xi \cdot x} \\
\left|\sum_{\xi \in \mathbb{Z}^{k-1} \backslash 0} e^{-\frac{\pi^{2}}{r}\left(\xi, \tilde{J}_{k}^{-1} \xi\right)} e^{2 \pi i \xi \cdot x}\right| \leq \sum_{\xi \in \mathbb{Z}^{k-1} \backslash 0}\left|e^{-\frac{\pi^{2}}{r}\left(\xi, \tilde{J}_{k}^{-1} \xi\right)}\right|
\end{gathered}
$$

Os autovalores de $\tilde{J}_{k-1}$ podem ser encontrados se notarmos que esse é o operador laplaciano discreto. Dessa forma os autovetores tem a forma

$$
\left(\begin{array}{c}
\sin \left(m \frac{\pi}{k}\right) \\
\sin \left(m \frac{\pi}{k} 2\right) \\
\vdots \\
\sin \left(m \frac{\pi}{k}(k-1)\right)
\end{array}\right)
$$

para $m \in \mathbb{Z}$. Aplicando $\tilde{J}_{k-1}$ a esse vetor obetoms, na i-ésima linha

$$
2 \sin \left(m \frac{\pi}{k} i\right)-\sin \left(m \frac{\pi}{k}(i-1)\right)-\sin \left(m \frac{\pi}{k}(i+1)\right)=2\left(1-\cos \left(\frac{m \pi}{k}\right)\right) \sin \left(m \frac{\pi}{k} i\right)
$$

Desse modo os autovalores da inversa são, maiores ou iguais a $\frac{1}{4}$. Assim

$$
\begin{gathered}
-\left(\xi, \tilde{J}_{k}^{-1} \xi\right) \geq-\frac{1}{4}\|\xi\| \\
\sum_{\xi \in \mathbb{Z}^{k-1} \backslash 0}\left|e^{-\frac{\pi^{2}}{r}\left(\xi, \tilde{J}_{k}^{-1} \xi\right)}\right| \leq \sum_{\xi \in \mathbb{Z}^{k-1} \backslash 0}\left|e^{-\frac{\pi^{2}}{4 r} \sum_{i=1}^{k-1} \xi_{i}^{2}}\right|=\left(2 \sum_{\xi_{i}=1}^{\infty} e^{-\frac{\pi^{2}}{4 r} \xi_{i}^{2}}\right)^{k-1}
\end{gathered}
$$

Outra coisa que notamos é que o termo convolutivo,

$$
\sqrt{\frac{\left(\pi / r\left(\beta^{\prime}\right)\right)^{k-1}}{k}} \sum_{\xi \in \mathbb{Z}^{k-1}} e^{-\frac{\pi^{2}}{r}\left(\xi, \tilde{J}_{k}^{-1} \xi\right)} e^{2 \pi i \xi \cdot x}
$$

oscila em torno do valor da convolução contínua.

\subsection{Limite de $\mathbf{r}$ pequeno}

Vamos analisar a equação de ponto fixo no limite em que $r$ é pequeno, ou seja, em que

$$
M_{n} \approx \sqrt{\frac{(\pi / r)^{k-1}}{k}}
$$




$$
e^{-r(\beta)(n-\bar{n})^{2}+b(\beta)(n-\bar{n})}=\frac{e^{-\beta\left(2^{d-2}-1\right)\left(\begin{array}{c}
n-\bar{n} \\
2
\end{array}\right)}}{k^{n}} \sqrt{\frac{(\pi / r)^{k-1}}{k}} e^{-\frac{r\left(\beta^{\prime}\right)}{k}(n-\bar{n})^{2}+b\left(\beta^{\prime}\right)(n-\bar{n})}
$$

Essa equação sugere que a normalização em cada escala deva ser

$$
C=N:=\sqrt{\frac{(\pi / r)^{k-1}}{k}}
$$

Temos assim exatamente o mesmo ponto fixo do limite contínuo. Com esse resultado percebemos que esse ponto fixo vale quando $\beta$ é pequeno, ou seja a altas temperaturas.

\subsection{Estabilidade do ponto fixo no limite de altas temperaturas}

Temos os seguintes mapas para os parâmetros $r$ e $b$, obtidos em 4.19):

$$
\left\{\begin{array}{l}
r^{(l)}(\beta)=\frac{\beta\left(2^{(d-2)}-1\right)}{2}+\frac{r^{(l+1)}\left(2^{d-2} \beta\right)}{2^{d}}=\frac{\beta\left(2^{(d-2)}-1\right)}{2}+\frac{r^{(l+1)}(\beta)}{4} \\
\left.b^{(l)}(\beta)=\frac{\beta\left(2^{(d-2)}-1\right)}{2}-d \log (2)+b^{(l+1)}\left(2^{d-2} \beta\right)\right)=\frac{\beta\left(2^{(d-2)}-1\right)}{2}-d \log (2)+b^{(l+1)}(\beta) \frac{k}{4}
\end{array}\right.
$$

Os respectivos mapas linearizados em torno do ponto fixo são

$$
\begin{gathered}
\left\{\begin{array}{l}
r^{(l)}-r^{*}=\left.\frac{d r^{(l)}}{d r^{(l+1)}}\right|_{r^{*}}\left(r^{(l+1)}-r^{*}\right) \\
b^{(l)}-b^{*}=\left.\frac{d b^{(l)}}{d b^{(l+1)}}\right|_{b^{*}}\left(b^{(l+1)}-b^{*}\right)
\end{array}\right. \\
\left\{\begin{array}{l}
r^{(l)}-r^{*}=\frac{1}{4}\left(r^{(l+1)}-r^{*}\right) \\
b^{(l)}-b^{*}=\frac{k}{4}\left(b^{(l+1)}-b^{*}\right)
\end{array}\right.
\end{gathered}
$$

como $\frac{1}{k}<1$ percebemos que o ponto fixo em $r$ é estável. No caso de $b, \frac{k}{4}>1$ para todas as dimensões $d \geq 3$, o que faz do ponto fixo instável para $b$. 



\section{Capítulo 5}

\section{A Matriz Q}

Existe também uma outra forma de representar as convoluções discretas. A fórmula de Poisson, apesar de ser boa no limite em que $\beta$ é pequeno, nem sempre apresenta bons resultados para $\beta$ grande. Dessa forma é vantajoso encontrar uma expressão sem o uso da fórmula de Poisson para as convoluções. Para isso simplesmente consideramos a equação (3.8)

$$
W(n, \bar{n}, \beta)=\frac{e^{-\beta\left(2^{d-2}-1\right)\left(\begin{array}{c}
n-\bar{n} \\
2
\end{array}\right)}}{k^{n}}\left(W\left(\cdot, \bar{n}_{1}, \beta^{\prime}\right) * \cdots * W\left(\cdot, \bar{n}_{k}, \beta^{\prime}\right)\right)(n)
$$

onde $\sum_{i=1}^{k} n_{i}=n$ e $\bar{n}_{i}$ é o background limitado a $D_{i}$, cujo $i$ indexa também $n_{i}$ e consideramos também o Ansatz Gaussiano

$$
W^{*}(n, \bar{n}, \beta)=e^{-r(\beta)(n-\bar{n})^{2}+b(\beta)(n-\bar{n})} .
$$

Assumimos agora que todos os $\bar{n}_{i}$ são multiplos de $k$. Isso quer dizer que o background sempre preenche todos os hipercubos de uma hierarquia com o mesmo número de partículas. Essa hipótese pode parecer restritiva demais, mas veremos em breve que ela não tira a generalidade de modo algum.

Com esse Ansatz a parte da convolução é uma soma de um produto de Gaussianas

$$
W^{*}\left(n_{1}, \frac{\bar{n}}{k}, \beta^{\prime}\right) \cdots W^{*}\left(n_{k}, \frac{\bar{n}}{k}, \beta^{\prime}\right)
$$

e podemos colocar em evidência uma gaussiana $G$ tal que

$$
G\left(n, \bar{n}, \beta^{\prime}\right)=W^{*}\left(n, \bar{n}, \beta^{\prime}\right)_{\frac{r}{k}}
$$

$\operatorname{com} \frac{r}{k}$ no lugar de $r$. Dessa forma

$$
W^{*}\left(n_{1}, \frac{\bar{n}}{k}, \beta^{\prime}\right) \cdots W^{*}\left(n_{k}, \frac{\bar{n}}{k}, \beta^{\prime}\right)=\frac{W^{*}\left(n_{1}, \frac{\bar{n}}{k}, \beta^{\prime}\right) \cdots W^{*}\left(n_{k}, \frac{\bar{n}}{k}, \beta^{\prime}\right)}{G\left(n, \bar{n}, \beta^{\prime}\right)} G\left(n, \bar{n}, \beta^{\prime}\right),
$$


o que resulta em

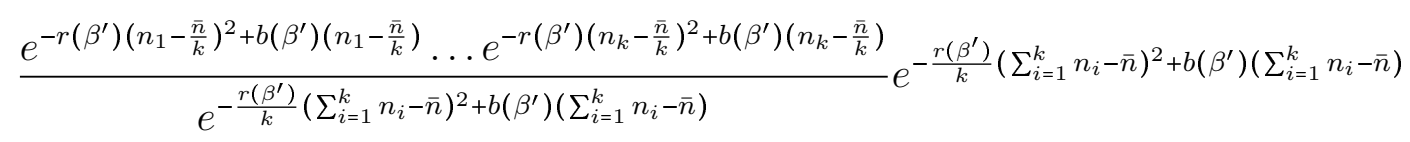

Nessa fração os termos com $b\left(\beta^{\prime}\right)$ se cancelam.

$$
\frac{e^{-r\left(\beta^{\prime}\right)\left(n_{1}-\frac{\bar{n}}{k}\right)^{2}+b\left(\beta^{\prime}\right)\left(n_{1}-\frac{\bar{n}}{k}\right)} \ldots e^{-r\left(\beta^{\prime}\right)\left(n_{k}-\frac{\bar{n}}{k}\right)^{2}+b\left(\beta^{\prime}\right)\left(n_{k}-\frac{\bar{n}}{k}\right)}}{e^{-\frac{r\left(\beta^{\prime}\right)}{k}\left(\sum_{i=1}^{k} n_{i}-\bar{n}\right)^{2}}}=e^{b\left(\beta^{\prime}\right)\left(n_{k}-\bar{n}\right)-r\left(\beta^{\prime}\right)\left(\left(1-\frac{1}{k}\right) \sum_{i=1}^{k} n_{i}^{2}-\frac{1}{k} \sum_{i \neq j}^{k} n_{i} n_{j}\right)}
$$

Com essa úlitma equação fica claro que podemos escrever a convolução de uma forma matricial. A função em 5.5 pode ser escrita como

$$
e^{b\left(\beta^{\prime}\right)\left(n_{k}-\bar{n}\right)-r\left(\beta^{\prime}\right)(\vec{n}, Q \vec{n})}
$$

onde

$$
Q:=\left(\begin{array}{ccccc}
\left(1-\frac{1}{k}\right) & -\frac{1}{k} & -\frac{1}{k} & \cdots & -\frac{1}{k} \\
-\frac{1}{k} & \left(1-\frac{1}{k}\right) & -\frac{1}{k} & \cdots & -\frac{1}{k} \\
\vdots & \ddots & & & \vdots \\
-\frac{1}{k} & \cdots & & -\frac{1}{k} & \left(1-\frac{1}{k}\right)
\end{array}\right)
$$

e

$$
\vec{n}:=\left(\begin{array}{c}
n_{1} \\
n_{2} \\
\vdots \\
n_{k}
\end{array}\right)
$$

Dessa forma ficamos com

$$
\begin{gathered}
W^{*}\left(n_{1}, \frac{\bar{n}}{k}, \beta^{\prime}\right) \cdots W^{*}\left(n_{k}, \frac{\bar{n}}{k}, \beta^{\prime}\right) \\
=e^{-\frac{r\left(\beta^{\prime}\right)}{k}(n-\bar{n})^{2}+b\left(\beta^{\prime}\right)(n-\bar{n})} \sum_{n_{1}=0}^{n} \cdots \sum_{n_{k-1}=0}^{n_{k-2}} e^{-r\left(\beta^{\prime}\right)(\vec{n}, Q \vec{n})}
\end{gathered}
$$

e a equação

$$
\begin{gathered}
e^{-r(\beta)(n-\bar{n})^{2}+b(\beta)(n-\bar{n})} \\
k^{n} e^{-\beta\left(\left(2^{(d-2)}-1\right)\left(\begin{array}{c}
n-\bar{n} \\
2
\end{array}\right)\right)} e^{-\frac{r\left(\beta^{\prime}\right)}{k}(n-\bar{n})^{2}+b\left(\beta^{\prime}\right)(n-\bar{n})} \sum_{\substack{n_{1}=0 \\
\sum_{i} n_{i}=n}}^{n} \ldots \sum_{\substack{n_{k-1}=0 \\
n}}^{n} e^{-r\left(\beta^{\prime}\right)(\vec{n}, Q \vec{n})}
\end{gathered}
$$

É importante notar que a matriz $Q$ é singular, ou seja, positiva semidefinida (pode-se ver que nenhum autovalor é negativo via discos de Gershgorin). Isso significa que a soma pode não convergir 
quando estendemos seus limites para $\infty$. Felizmente, a matriz aparece somente no contexto de uma convolução e a convolução fixa a soma das componentes do vetor $\vec{n}$ sobre o qual $Q$ opera. O posto da matriz $Q$ é $k-1$, desse modo só há um autovetor correspondente a zero. Esse vetor é da forma $\vec{\lambda}:=(a, \ldots, a)$, mas como a soma dos elementos está fixa, temos que $\vec{\lambda}=(1 / k, \ldots, 1 / k)$, significando que ele está fixo e a soma não ocorrerá nessa direção. Por conta disso a matriz $Q$ é definida somente no complemento ortogonal de $\vec{\lambda}$, sendo que a função é somável (e integrável) nesse espaço.

A matriz $Q_{k \times k}$ é também uma matriz circulante, isso significa que seu $l$-ésimo autovetor normalizado, $0 \leq l \leq k-1$, tem a forma

$$
\vec{\lambda}_{l}=\frac{1}{\sqrt{k}}\left(\begin{array}{c}
1 \\
e^{\frac{2 \pi i}{k} 1 l} \\
\vdots \\
e^{\frac{2 \pi i}{k}(k-1) l}
\end{array}\right)
$$

e os autovalores são $\lambda_{0}=0$ e $\lambda_{l}=1$.

\subsection{O Limite de $n$ grande em $Q$}

Queremos agora encontrar uma equação de ponto fixo para todas as temperaturas, no limite de $n$ grande. Já sabemos que no caso do Ansatz Gaussiano, uma gaussiana é levada em funções que oscilam. Desse modo vamos realizar um Ansatz ligeiramente diferente nesse caso mais geral. Inspirados pelos resultados anteriores, vamos assumir

$$
W(n, \bar{n}, \beta)=V(n) e^{-r(\beta)(n-\bar{n})^{2}}
$$

Onde $V(n)$ é uma função periódica de período $k$ e limitada. Assumimos também que o número $n$ de partículas é múltiplo de $k$.

$$
\begin{gathered}
W(n, \bar{n}, \beta)=\frac{e^{-\beta\left(2^{d-2}-1\right)\left(\begin{array}{c}
n-\bar{n} \\
2
\end{array}\right)}}{k^{n}}\left(W\left(\cdot, \bar{n}_{1}, \beta^{\prime}\right) * \cdots * W\left(\cdot, \bar{n}_{k}, \beta^{\prime}\right)\right)(n), \\
=\frac{e^{-\beta\left(\left(2^{(d-2)}-1\right)\left(\begin{array}{c}
n-\bar{n} \\
2
\end{array}\right)\right)}}{k^{n}} e^{-\frac{r\left(\beta^{\prime}\right)}{k}(n-\bar{n})^{2}+b\left(\beta^{\prime}\right)(n-\bar{n})} \sum_{\substack{n_{1}=0 \\
\sum n_{i}=n}}^{\infty} \ldots \sum_{n_{k}=0}^{\infty} V\left(n_{1}\right) \ldots V\left(n_{k}\right) e^{-r\left(\beta^{\prime}\right)(\vec{n}, Q \vec{n})}
\end{gathered}
$$

Para tomar o limite de $n \rightarrow \infty$, realizamos uma mudança de variáveis na soma $n_{i} \rightarrow \frac{n_{i}}{n}$. Definimos ainda as variáveis

$$
\begin{aligned}
& y_{i}:=\frac{n_{i}}{N} \\
& x:=\frac{n}{N},
\end{aligned}
$$

onde $N$ é um inteiro cujo limite tomaremos para infinito junto com $n$, de modo que $x$ permaneça em um valor fixo. 


$$
\sum_{\substack{n_{1}=0 \\ \sum n_{i}=n}}^{\infty} \ldots \sum_{\substack{n_{k}=0 \\ \infty}}^{\infty} V\left(n_{1}\right) \ldots V\left(n_{k}\right) e^{-r\left(\beta^{\prime}\right)(\vec{n}, Q \vec{n})}=\sum_{\substack{y_{1}=0 \\ \sum y_{i}=x}}^{\infty} \ldots \sum_{\substack{y_{k}=0 \\ \infty}}^{\infty} V\left(y_{1} N\right) \ldots V\left(y_{k} N\right) e^{-N^{2} r\left(\beta^{\prime}\right)(\vec{y}, Q \vec{y})} \frac{1}{N^{k-1}} N^{k-1}
$$

onde $\vec{y}=\left(y_{1}, \ldots, y_{k}\right)$

Sabemos que o operador $Q$ tem todos os seus autovalores positivos, exceto um que é 0 e sabemos que um autovetor constante tem autovalor correspondente zero.

Dada a definição de uma distribuição delta de Dirac (Olhar nos apêndices B), gostaríamos de provar que a sequência de funções $\left(k_{N}\right)_{N \in \mathbb{N}}$

$$
k_{N}(\vec{y}):=e^{-N^{2} r\left(\beta^{\prime}\right)(\vec{y}, Q \vec{y})} C
$$

onde $C$ é uma constante de normalização, satisfaz as propriedades listadas no apêndice B

1. $k_{n}(\vec{x}) \geq 0$

2. $\int \cdots \int_{\mathbb{R}^{d}} k_{N}(\vec{x}) d \vec{x}=1$

3. dados $\varepsilon>0$ e $\eta>0$, então existe $N_{0} \leq N$ tal que

$$
\int \cdots \int_{|\vec{x}|>\eta} k_{n}(\vec{x}) d \vec{x}<\varepsilon
$$

para que possamos definir uma distribuição delta.

A primeira propriedade é trivial para a função $k_{N}(\vec{y})$, lembrando que a matriz $Q$ está definida somente no complemento ortogonal de $\vec{\lambda}$.

A segunda propriedade pode ser demonstrada percebendo que a escolha de uma constante $C$ é possível pela integral ser limitada no complemento ortogonal de $\vec{\lambda}$.

$$
\begin{gathered}
\int_{-\infty}^{\infty} \cdots \int_{-\infty}^{\infty} e^{-N^{2} r\left(\beta^{\prime}\right)\left(\left(x-y_{1}\right)^{2}+\sum_{i=1}^{k-2}\left(y_{i}-y_{i+1}\right)^{2}+y_{k-1}^{2}-\frac{1}{k} x^{2}\right)} C d y_{1} \ldots d y_{k-1}=\sqrt{\frac{\pi^{k-1}}{r^{k-1} k}} \frac{C}{N^{k-1}}=1 \\
C=\sqrt{\frac{k r^{k-1}}{\pi^{k-1}}} N^{k-1}
\end{gathered}
$$

A terceira pode ser provada se notarmos que

$$
\int \cdots \int_{|\vec{x}|>\eta} k_{n}(\vec{x}) d \vec{x}=C \int \cdots \int_{|\vec{y}|>\eta} \delta\left(x-\sum_{i=1}^{k} y_{i}\right) e^{-N^{2} r\left(\sum_{i=1}^{k} y_{i}^{2}-\frac{x^{2}}{k}\right)} d \vec{y}
$$


notamos também que se $N_{1}<N_{2}$ então

$$
e^{-N_{1}^{2} r\left(\sum_{i=1}^{k} y_{i}^{2}-\frac{x^{2}}{k}\right)}>e^{-N_{2}^{2} r\left(\sum_{i=1}^{k} y_{i}^{2}-\frac{x^{2}}{k}\right)}
$$

exceto quando $\sum_{i=1}^{k} y_{i}^{2}-\frac{x^{2}}{k}=0$, em que as duas funções seriam iguais.

Sabemos que a integral da função em todo o espaço é 1 . Desse modo, se a integral for limitada em uma região $|\vec{x}|>\eta$, então ela será menor que 1 . Se escolhermos $N$ grande o suficiente, a função dentro da integral diminuirá, tendendo a zero em quase todos os pontos. Isso significa que para qualquer $\eta$ podemos aumentar $N$ até um ponto em que $\int \cdots \int_{|\vec{x}|>\eta} k_{n}(\vec{x}) d \vec{x}$ fique menor que uma constante $\varepsilon$

Dessa forma, no limite $N \rightarrow \infty$ temos que

$$
\lim _{N \rightarrow \infty} e^{-N^{2} r\left(\beta^{\prime}\right)(\vec{y}, Q \vec{y})} N^{k-1}=\sqrt{\frac{\pi^{k-1}}{r^{k-1} k}} \delta\left(y_{1}-\frac{1}{k}\right) \ldots \delta\left(y_{k}-\frac{1}{k}\right)
$$

onde $\vec{\lambda}=\left(\frac{1}{k}, \ldots, \frac{1}{k}\right)$ é o autovetor correspondente ao autovalor zero. Desse modo

$$
\begin{gathered}
\lim _{\substack{N \rightarrow \infty \\
y_{1}=0}} \sum_{\substack{\sum y_{i}=x \\
y_{k}=0}}^{\infty} N\left(y_{1} N\right) \ldots V\left(y_{k} N\right) e^{-N^{2} r\left(\beta^{\prime}\right)(\vec{y}, Q \vec{y})} \frac{1}{N^{k-1}} N^{k-1} \\
=\int_{\substack{\mathbb{R}^{k} \\
\sum_{i}=1}}^{\infty} V\left(y_{1} N\right) \ldots V\left(y_{k} N\right) \sqrt{\frac{\pi^{k-1}}{r^{k-1} k}} \delta\left(y_{1}-\frac{1}{k}\right) \ldots \delta\left(y_{k}-\frac{1}{k}\right) d^{k} \vec{x}=V^{k}\left(\frac{n}{k}\right) \sqrt{\frac{\pi^{k-1}}{r^{k-1} k}} \\
V(n) e^{-r(\beta)(n-\bar{n})^{2}+b(\beta)(n-\bar{n})}=\frac{e^{-\beta\left(\left(2^{(d-2)}-1\right)\left(\begin{array}{c}
n-\bar{n} \\
2
\end{array}\right)\right)}}{k^{n} C} e^{-\frac{r\left(\beta^{\prime}\right)}{k}(n-\bar{n})^{2}+b\left(\beta^{\prime}\right)(n-\bar{n})} V^{k}\left(\frac{n}{k}\right) \sqrt{\frac{\pi^{k-1}}{r^{k-1} k}}
\end{gathered}
$$

e o que isso significa? Que a transformação de GR leva funções da forma Gaussiana em $n$ vezes algo periódico em funções da mesma forma.

O problema é que tudo isso foi feito em uma subsequência de $n$ em que os valores de $n$ são múltiplos de $k$. O que acontece para o caso em que $n=k\left[\frac{n}{k}\right]+\alpha$ tem um digito $\alpha \neq 0$ ?

Nesse caso o vetor $\vec{y}$ nunca vai poder assumir o valor $\vec{\lambda}$, pois existirão $\alpha$ particulas a mais do que o necessário para uma distribuição uniforme. O mais próximo que $\vec{y}$ pode ficar de $\vec{\lambda}$ é quando $\alpha$ de suas componentes forem $\left[\frac{n}{k}\right]+1$ e as outras $k-\alpha$ forem $\left[\frac{n}{k}\right]$. 



\section{Capítulo 6}

\section{Discussão final e próximos passos}

Com esse trabalho conseguimos formular o modelo contínuo de partículas do gás Coulombiano hierárquico de duas espécies, uma dessas mantida fixa no estado fundamental, no contexto de grupos de renormalização. Essa formulação nos ajudou a perceber a forma que a função de partição deve ter, primeiramente com as convoluções (já que convoluções de Gaussianas resultam em Gaussianas) e depois com a análise do caso limite em que $r$ é pequeno e corrobora a hipótese.

O fenômeno que mais se sobressai em toda a discussão anterior é, provavelmente, o das oscilações na função de partição do sistema. Além disso, temos a aproximação das convoluções discretas por contínuas, que funciona surpreendentemente bem e dão um valor em torno do qual as oscilações ocorrem. Isso indica que o que gera as oscilações, é o fato de o modelo ser de partículas discretas. A adição de partículas em partições específicas, sem preencher todas as partições de uma hierarquia igualmente, gera as oscilações conforme existem diferentes números de combinações em que as partículas conseguem se ajustar no sistema.

Fica faltando ainda encontrar, de fato, a função de partição para o caso geral e fazer a análise de sua estabilidade. Existe uma proposta de qual o ponto fixo deve ser, mas é necessário ainda um pouco mais de estudo antes de uma formulação definitiva. Tendo o ponto fixo, poderemos fazer a análise de sua estabilidade. Imaginamos, pelo caso limite discutido aqui, que o ponto fixo vá ser estável. Isso significará que, mesmo olhando nas menores hierarquias uma distribuição que não segue a forma $G(n) V(n)(G(n)$ é uma gaussiana e $V(n)$ é uma função que oscila), depois de suficientes iterações do mapa, a distribuição chegará na forma $G(n) V(n)$. Com isso saberemos o comportamento típico de uma hierarquia e assim poderemos passar para a ideia que motivou toda a pesquisa: caracterizar a rigidez do sistema.

Tendo a distribuição de probabilidades do sistema, podemos finalmente usar estimativas, para caracterizarmos a variância do sistema, e assim esperamos mostrar rigorosamente que se trata de um sistema rígido. 

Apêndices 



\section{Apêndice A}

\section{Integrais}

Duas integrais são fundamentais para a obtenção dos nossos resultados.

A primeira dessas integrais é

$$
\int_{D_{0}} \omega(x, y) d^{d} y
$$

e a segunda é

$$
\int_{D_{0}} \int_{D_{0}} \omega(x, y) d^{d} x d^{d} y
$$

onde $\omega(x, y)$ é o potencial Coulombiano hierárquico dado pela Definição 1 .

O primeiro fato que notamos para calcular A.1) é que, para um dado $x$, o conjunto de todos os $y \operatorname{com} \omega(x, y)=2^{(d-2) k}$ é exatamente a união de todos os membros de $\mathcal{D}_{k} \subset D_{k-1}$, exceto aquele que contém $x$. O que corresponde a $2^{d}-1$ hipercubos $D_{k} \in \mathcal{D}_{k}$ indexados $D_{k, i} \operatorname{com} 1 \leq i \leq 2^{d}-1 \mathrm{e}$ $D_{k, x} \in \mathcal{D}_{k}$ sendo o sub-hipercubo que contém $x$. A medida de Lebesgue de cada um desses elementos é $2^{-k d}$, o que significa que a medida de Lebesgue do conjunto é $2^{(1-k) d}-2^{-k d}$.

Para computar a integral A.1] vamos integrar separadamente nas regiões em que o potencial é constante, ou seja, integramos em todos os sub-hipercubos $D_{1, i}$ que não contém $x$, o que corresponde a $D_{0} \backslash D_{1, x}$. Nessa região o potencial é constante $2^{(d-2) 1}$. Depois integramos em todos os subhipercubos $D_{2, i} \in D_{1, x}$ que não contém $x$, o que corresponde a $D_{1, x} \backslash D_{2, x}$ e nessa região o potencial é $2^{(d-2) 2}$ e assim por diante.

$$
\begin{aligned}
\int_{D_{0}} \omega(x, y) d^{d} y & =\int_{D_{0} \backslash D_{1, x}} \omega(x, y) d^{d} y+\int_{D_{1, x} \backslash D_{2, x}} \omega(x, y) d^{d} y+\ldots \\
& =2^{(d-2) 1}\left(2^{0 . d}-2^{-d}\right)+2^{(d-2) 2}\left(2^{-d}-2^{-2 d}\right)+\cdots=\frac{2^{d}-1}{3}
\end{aligned}
$$

Disso segue a integral (A.2)

$$
\int_{D_{0}} \int_{D_{0}} \omega(x, y) d^{d} y d^{d} x=\int_{D_{0}} \frac{2^{d}-1}{3} d^{d} x=\frac{2^{d}-1}{3}
$$





\section{Apêndice B}

\section{Delta de Dirac}

A definição de uma função delta de Dirac pode ser feita generalizando a definição do livro do Djairo [5]:

Dada uma sequência de funções $k_{n}: \mathbb{R}^{d} \mapsto \mathbb{R}$ com as seguintes propriedades

1. $k_{n}(\vec{x}) \geq 0$

2. $\int \cdots \int_{\mathbb{R}^{d}} k_{n}(\vec{x}) d \vec{x}=1$

3. dados $\varepsilon>0$ e $\eta>0$, então existe $n_{0} \leq n$ tal que

$$
\int \cdots \int_{|\vec{x}|>\eta} k_{n}(\vec{x}) d \vec{x}<\varepsilon
$$

Entao definimos

$$
\lim _{n \rightarrow \infty} \int \cdots \int_{R^{d}} k_{n}(\vec{x}) \psi(\vec{x}) d \vec{x}=\int \cdots \int_{d^{d}} \delta(\vec{x}) \psi(\vec{x}) d \vec{x}=\psi(0)
$$

onde

$$
\delta(\vec{x})= \begin{cases}0 & \text { se } \vec{x} \neq 0 \\ \infty & \text { se } \vec{x}=0\end{cases}
$$

e

$$
\int \cdots \int_{R d} \delta(\vec{x}) d \vec{x}=1
$$

A distribuição (B.3), que satisfaz as propriedades 1-3, é chamada distribuição delta de Dirac. 



\section{Apêndice C}

\section{Fórmula de Poisson}

Para discutir a generalização multidimensional da fórmula da somatória de Poisson, seguimos o livro Introduction to Fourier Analysis on Euclidean Spaces de Elias M. Stein e Guido Weiss [14].

Definimos aqui $\Lambda=\mathbb{Z}^{n}$ como sendo uma rede homogênea de pontos com espaçamento unitário em $\mathbb{R}^{n}$ e $Q(m)$ como sendo um hipercubo de lado igual a 1 (definido sempre como aberto em um lado e fechado no oposto) e centrado no ponto $m \in \Lambda$.

O teorema que queremos provar é

Teorema 3. Suponha que $f \in L^{1}$. Então a série $\sum_{m \in \Lambda} f(x+m)$ converge na norma de $L^{1}$. A função resultante tem a seguinte expansão de Fourier

$$
\begin{gathered}
\sum_{m \in \Lambda} \hat{f}(m) e^{2 \pi i x \cdot m} \\
\hat{f}(y):=\int_{\mathbb{R}^{n}} f(x) e^{-2 \pi i y \cdot x} d^{n} x
\end{gathered}
$$

Onde $L^{1}$ denota o espaço de funções que são Lebesgue-integráveis em valor absoluto e onde funções que coincidem em quase toda parte são identificadas.

\section{Prova do Teorema 3.}

$$
\int_{Q(0)}\left|\sum_{m \in \Lambda} f(x+m)\right| d^{n} x \leq \sum_{m \in \Lambda} \int_{Q(0)}|f(x+m)| d^{n} x=\sum_{m \in \Lambda} \int_{Q(m)}|f(x)| d^{n} x=\int_{\mathbb{R}^{n}}|f(x)| d^{n} x<\infty
$$

Isso mostra que a série $\sum_{m \in \Lambda} f(x+m)$ converge em valor absoluto na norma de $L^{1}$. Usando uma troca similar de somatória e integral, podemos avaliar agora os coeficientes de Fourier de $F(x):=\sum_{m \in \Lambda} f(x+m)$ :

$$
\hat{F}(m)=\int_{Q(0)}\left(\sum_{m^{\prime} \in \Lambda} f\left(x+m^{\prime}\right)\right) e^{-2 \pi i m \cdot x} d^{n} x=\sum_{m^{\prime} \in \Lambda} \int_{Q(0)} f\left(x+m^{\prime}\right) e^{-2 \pi i m \cdot x} d^{n} x
$$




$$
=\sum_{m^{\prime} \in \Lambda} \int_{Q\left(m^{\prime}\right)} f(x) e^{-2 \pi i m \cdot x} d^{n} x=\int_{\mathbb{R}^{n}} f(x) e^{-2 \pi i m \cdot x} d^{n} x=\hat{f}(m)
$$

Isso conclui a prova e assim temos a forma da expansão de Fourier.

Corolário 1. Suponha que $F \in L^{1} e \sum_{m \in \Lambda}\left|a_{m}\right|<\infty$, onde $\left\{a_{m}\right\}$ são os coeficientes de Fourier de $F$. Então $F$ pode ser modificada em um conjunto de medida nula, de modo que ela seja $C$, mas ainda assim seja igual a $\sum_{m \in \Lambda} a_{m} e^{2 \pi i m \cdot x}$ para todo $x$.

Prova do Corolário 1. A prova desse corolário não cabe no escopo do apêndice, mas pode ser encontrada em [14].

Para termos a fórmula do somatório de Poisson provamos o seguinte Corolário:

Corolário 2. Supomos que

$$
\hat{f}(y)=\int_{\mathbb{R}^{n}} f(x) e^{-2 \pi i x \cdot y} d^{n} x
$$

$e$

$$
f(x)=\int_{\mathbb{R}^{n}} \hat{f}(y) e^{2 \pi i x \cdot y} d^{n} y
$$

e que tanto $|f(x)|$ quanto $|\hat{f}(x)|$ tenham decaimento mais rápido que qualquer potência, além de continuidade para $f$ e $\hat{f}$. Então

$$
\sum_{m \in \Lambda} f(x+m)=\sum_{m \in \Lambda} \hat{f}(m) e^{2 \pi i m \cdot x} .
$$

Além disso, a série da direita converge absolutamente.

Prova do Corolário 2. Pelo que assumimos em $\hat{f}$, a série de Fourier da direita converge absolutamente.

De acordo com o corolário (1) e o teorema anterior, $F(x):=\sum_{m \in \Lambda} f(x+m)$ pode ser modificada em conjunto de medida nula para ter a série de Fourier $\sum_{m \in \Lambda} \hat{f}(m) e^{2 \pi i m \cdot x}$ e ser igual a ela em todo ponto. Pelo que assumimos sobre o decaimento das funções, as séries são uniformemente convergentes e as somas têm como termos, funções contínuas. Com isso a igualdade do corolário tem que valer para todo $x$. 


\section{Referências Bibliográficas}

[1] GUIDI, Leonardo F; MARCHETTI, Domingos H. U. Renormalization group flow on the two-dimensional hierarchical Coulomb gas. . Communications in Mathematical Physics, New York, Springer-Verlag, v. 219, n. 3, p. 671-702, 2001.

[2] MARCHETTI, D. H. U.; PEREZ, J. Fernando. The Kosterlitz-Thouless phase transition in two-dimensional hierarchical Coulomb gases Journal of Statistical Physics volume 55, 141-156, 1989

[3] MARCHETTI, D. H. U. Transição de Kosterlitz-Thouless em Modelos Hierárquicos: Uma Análise Rigorosa Via Grupo de Renormalização. Tese (Doutorado em Física) - Instituto de Física da Universidade de São Paulo, 1988

[4] JANCOVICI, B.; LEBOWITZ, J. L.; MAGNIFICAT, G. Large charge fluctuations in classical Coulomb systems. Journal of Statistical Physics volume 72, 773-787, 1993

[5] FIGUEIREDO, Djairo G. Análise de Fourier e Equações Diferenciais Parciais. IMPA, 2018, ISBN: 9788524404283.

[6] CHATTERJEE, Sourav. Rigidity of the three-dimensional hierarchical Coulomb gas. Probability Theory and Related Fields 175, 1123-1176, 2019

[7] Felder, Giovanni. Renormalization group in the local potential approximation.. Comm. Math. Phys. 111 (1987), no. 1, 101-121. https://projecteuclid.org/euclid.cmp/1104159469

[8] GANGULY, Shirshendu; SARKAR, Sourav. Ground states and hyperuniformity of the hierarchical Coulomb gas in all dimensions. Probability Theory and Related Fields 177, 621-675, 2020

[9] DA FONSECA, C.M.; PETRONILHO, J.. Explicit inverses of some tridiagonal matrices. Linear Algebra and its Applications, 325 (2001), 7-21 https://doi.org/10.1016/S0024-3795(00)00289-5

[10] GINIBRE, J. Rigorous Lower Bound on the Compressibility of a Classical System. Physics Letters, Volume 24A, number 4, 1967

[11] TORQUATO, Salvatore; STILLINGER, Frank H. Local density fluctuations, hyperuniformity, and order metrics Physical Review E 68, 041113, 2003

[12] COSTIN, Ovidiu; LEBOWITZ, Joel L. Gaussian Fluctuation in Random Matrices Phys. Rev. Lett. 75, 69,1995 
[13] GHOSH, Subhroshekhar; LEBOWITZ, Joel L. Fluctuations, Large Deviations and Rigidity in Hyperuniform Systems: A Brief Survey Indian J. Pure Appl. Math., 48(4): 609-631, 2017

[14] STEIN, Elias; WEISS, Guido. Introduction to Fourier Analysis on Euclidean Spaces. Princeton University Press, 1971. ISBN: 978-0-691-08078-9 